\title{
High Performance Multivariate Geospatial Statistics on Manycore Systems
}

\author{
Mary Lai O. Salvaña, Sameh Abdulah, Huang Huang, Hatem Ltaief, \\ Ying Sun, Marc G. Genton, and David E. Keyes
}

\begin{abstract}
Modeling and inferring spatial relationships and predicting missing values of environmental data are some of the main tasks of geospatial statisticians. These routine tasks are accomplished using multivariate geospatial models and the cokriging technique. The latter requires the evaluation of the expensive Gaussian log-likelihood function, which has impeded the adoption of multivariate geospatial models for large multivariate spatial datasets. However, this large-scale cokriging challenge provides a fertile ground for supercomputing implementations for the geospatial statistics community as it is paramount to scale computational capability to match the growth in environmental data coming from the widespread use of different data collection technologies. In this paper, we develop and deploy large-scale multivariate spatial modeling and inference on parallel hardware architectures. To tackle the increasing complexity in matrix operations and the massive concurrency in parallel systems, we leverage low-rank matrix approximation techniques with task-based programming models and schedule the asynchronous computational tasks using a dynamic runtime system. The proposed framework provides both the dense and the approximated computations of the Gaussian log-likelihood function. It demonstrates accuracy robustness and performance scalability on a variety of computer systems. Using both synthetic and real datasets, the low-rank matrix approximation shows better performance compared to exact computation, while preserving the application requirements in both parameter estimation and prediction accuracy. We also propose a novel algorithm to assess the prediction accuracy after the online parameter estimation. The algorithm quantifies prediction performance and provides a benchmark for measuring the efficiency and accuracy of several approximation techniques in multivariate spatial modeling.
\end{abstract}

Index Terms-Gaussian log-likelihood, geospatial statistics, high-performance computing, large multivariate spatial data, low-rank approximation, multivariate modeling/prediction.

\section{INTRODUCTION}

$\mathrm{T}$ HE convergence of high-performance computing (HPC) and big data brings great promise in accelerating and improving large-scale applications [1], [2] on climate and weather modeling [3], astronomy [4], transportation [5], and bioinformatics $|\overline{6}|$. Climate and weather modeling, in particular, is one of the first applications of HPC for big data |7|. The need to improve climate and weather models has pushed for advances in environmental data collection technologies such as spaceborne, airborne, and ground sensors [8]. The volume of data coming from these sources is huge and increasing. For instance, NASA's Earth Observing System Data and Information System (EOSDIS) is expected to archive more than 37 petabytes of data in 2020 [9]. By 2022 , the yearly increase is projected at 47.7 petabytes.

Environmental data, such as climate and weather variables, are often recorded from different spatial locations, and thus indexed by $\mathbf{s} \in \mathbb{R}^{d}, d \geq 1$, where $\mathbf{s}$ is the location of the measurement. Usually, there are multiple variables measured at each location, such as temperature, humidity, wind speed, and atmospheric pressure. These colocated variables may or may not depend on each other and on the variables at other locations.

- The authors are with the Extreme Computing Research Center, Computer, Electrical, and Mathematical Sciences and Engineering Division (CEMSE), King Abdullah University of Science and Technology (KAUST), Thuwal 23955-6900, Saudi Arabia. E-mail: \{Marylai.Salvana, Sameh.Abdulah, Huang.Huang, Hatem.Ltaief, Ying.Sun, Marc.Genton, David.Keyes\}@kaust.edu.sa.
Exposing spatial relationships among spatially referenced data can be accomplished using geographical information systems (GIS) [10]. Through GIS, one can produce scientific visualizations such as maps of raw data and spatial patterns, thereby facilitating exploratory data analysis, statistical analysis, and hypothesis testing. The big data era brought new challenges to GIS and its ability to process and analyze huge streams and volumes of geospatial data. However, the influx of big geospatial data were met with brand new capabilities of GIS made possible by HPC [11]. HPC boosts GIS operations and computations in the face of large amounts of geospatial data by utilizing modern hardware architectures such as computer clusters, GPUs, and cloud computing infrastructures [12], [13], [14], [15]. For instance, in [16], GPU accelerators have been used to accelerate the visualization of large-scale geospatial data. In [13], distributed GPU systems through Message Passing Interface (MPI) over Network of Workstations (NoW) and Compute Unified Device Architecture (CUDA) have been used to perform real-time map matching and slope computations of a large global positioning system (GPS) data. Another example has been shown in [17] where a graphbased methodology on a cluster computing paradigm was employed in land use/land cover change (LUCC) analysis; see [18] for other HPC-based GIS implementations review.

Aside from processing a huge amount of data harvested from different sources such as satellite images, model simulations, sensors, and the Internet of Things, a major concern when dealing with environmental datasets is missing data on one or a few variables. For instance, when 
using environmental variables as inputs to climate and weather models, the gaps in areas with no measurements caused by poor atmospheric conditions or defective sensors, to name a few, need to be filled [19], |20|. Several missing data interpolation techniques exist in the literature including numerical models, machine learning and deep learning models, and geospatial statistics models [21], |22], [23], [24], [25]. Numerical models solve a complex set of partial differential equations and generate large volumes of predictions on the quantities of interest, such as the concentrations of pollutants in the atmosphere [26], [27]. The strength in using numerical models in prediction lies in their physically consistent representations of the phenomena or systems being analyzed. However, numerical models cannot accurately predict on very fine spatial resolutions [28]. Machine learning and deep learning models capture the spatial relationships among environmental variables through feature representation learning using neural networks [29]. Image inpainting with transfer learning has been used to impute missing global surface temperature measurements in HadCRUT4 [30]. Generative adversarial network (GAN) was used in [31] to reconstruct the missing sea surface temperature readings in satellite images due to cloud disturbances. Several other machine learning and deep learning solutions to the missing data problem are listed in [28]. Although machine learning and deep learning have shown high prediction capabilities, they are devoid of physical knowledge of the process being modeled and predicted |25|. Furthermore, they suffer the drawback of being unable to describe explicitly the spatial relationship among environmental variables [32], i.e., absence of interpretability.

In this work, we adopt the multivariate geospatial statistical approach due to its ability to characterize the dependence structure of the underlying spatial data. The geospatial statistical models are also very easy to interpret and yield very high prediction accuracy. Multivariate geospatial statistics can interpolate environmental variables at unsampled locations by modeling the multivariate spatial dependencies using a multivariate covariance function, whose parameters are calibrated with the aid of a loglikelihood function. While every variable of interest can be modeled and predicted separately, it has been shown that more accurate predictions can be produced when modeling dependent variables jointly [33], |34].

Modeling the variables as realizations from a multivariate Gaussian random field is the cornerstone of multivariate geospatial statistics. Multivariate random fields are the equivalent of multivariate random variables, where a vector of variables is measured at each location [35]. Mathematically, this means that at location $\mathbf{s} \in \mathbb{R}^{d}, \overline{d \geq 1}$, each variable is considered as one component of the $p$-dimensional vector $\mathbf{Z}(\mathbf{s})$, i.e., $\mathbf{Z}(\mathbf{s})=\left\{Z_{1}(\mathbf{s}), \ldots, Z_{p}(\mathbf{s})\right\}^{\top}$, where $\top$ is the transpose operator, $p$ is the number of variables, and $Z_{i}(\mathbf{s}) \in \mathbb{R}$ indicates the value of the $i$ th variable at location $\mathbf{s}, i=1, \ldots, p$. When $\mathbf{Z}(\mathbf{s})$ is Gaussian, it is completely characterized by its mean vector and multivariate covariance function, which is more commonly known as crosscovariance function in geospatial statistics and multivariate kernel in computer science.

Cokriging is the prediction of multiple variables using an optimal predictor [33], [34]. To perform cokriging on a multivariate Gaussian random field, one only needs to specify a mean vector and a cross-covariance function. In this work, we assume that the multivariate Gaussian random field has mean zero and focus our attention on the cross-covariance function, which introduces considerable computational challenges. This zero-mean assumption is reasonable since regression can be used to remove the mean. Furthermore, we restrict our attention to a specific crosscovariance function that is stationary and isotropic. These assumptions on the cross-covariance function are building blocks to more sophisticated ones including nonstationary and anisotropic cross-covariance functions, which can be readily accommodated by our proposed framework. They do not present significant limitations on the large-scale multivariate geospatial analysis aimed at this work.

In practice, a class of cross-covariance functions is first selected and its unknown parameters are estimated from the data [33], [36], [37]. Estimation relies on the maximum likelihood estimation (MLE) approach [38], [39]. Suppose $\boldsymbol{\theta} \in \mathbb{R}^{q}$ collects all the $q$ true unknown parameters of the cross-covariance function. The MLE of $\boldsymbol{\theta}$, denoted by $\widehat{\boldsymbol{\theta}} \in$ $\mathbb{R}^{q}$, is the $q$-dimensional vector which maximizes the loglikelihood function

$$
l(\boldsymbol{\theta})=-\frac{n p}{2} \log (2 \pi)-\frac{1}{2} \log |\boldsymbol{\Sigma}(\boldsymbol{\theta})|-\frac{1}{2} \mathbf{Z}^{\top} \boldsymbol{\Sigma}(\boldsymbol{\theta})^{-1} \mathbf{Z},
$$

with respect to all $q$ parameters in $\boldsymbol{\theta}$. Here $\mathbf{Z} \in \mathbb{R}^{p n}$ collects all the $p$-dimensional vectors $\mathbf{Z}(\mathbf{s})$ at $n$ locations, $\mathbf{s}_{1}, \mathbf{s}_{2}, \ldots, \mathbf{s}_{n}$, i.e., $\mathbf{Z}=\left\{\mathbf{Z}\left(\mathbf{s}_{1}\right)^{\top}, \mathbf{Z}\left(\mathbf{s}_{2}\right)^{\top}, \ldots, \mathbf{Z}\left(\mathbf{s}_{n}\right)^{\top}\right\}^{\top}$. $\boldsymbol{\Sigma}(\boldsymbol{\theta})$ is the $p n \times p n$ cross-covariance matrix for $\mathbf{Z}$ and $|\boldsymbol{\Sigma}(\boldsymbol{\theta})|$ denotes the determinant of $\boldsymbol{\Sigma}(\boldsymbol{\theta})$. The entries of $\boldsymbol{\Sigma}(\boldsymbol{\theta})$ are calculated from the cross-covariance function that is known up to $\boldsymbol{\theta}$. The procedure in constructing this cross-covariance matrix is discussed in more detail in Section 5.2

The MLE involves the computation of the log-likelihood function in Equation (1) for each iteration in the optimization. In large-scale multivariate problems with irregularly spaced locations, the $\log$-likelihood requires $\mathcal{O}\left(p^{2} n^{2}\right)$ memory and $\mathcal{O}\left(p^{3} n^{3}\right)$ operations per iteration, due to the Cholesky decomposition of $\boldsymbol{\Sigma}(\boldsymbol{\theta})$. The prohibitive computational cost and corresponding storage of computing the log-likelihood function can be reduced by relying on lowrank approximation techniques that exploit the low-rank representations of the cross-covariance matrix, for instance, the Tile Low-Rank (TLR) approximation [40], [41], [42]. TLR is the preferred approximation approach in case of parallel execution and it involves dividing the matrix into a set of tiles, then applying low-rank approximation separately to each tile.

The quality of the predictions can be assessed using the mean square prediction error (MSPE) |42] and the mean square relative prediction error (MSRP) [43]. However, it has been shown that these commonly used criteria cannot adequately assess the prediction efficiency when different approximation methods are involved and are up for comparisons |44|. As an alternative, under univariate modeling, the authors in [44] proposed two criteria, namely, the mean loss of efficiency (MLOE) and the mean misspecification of the mean square error (MMOM), to more appropriately assess the loss of prediction efficiency when an approximated version of the model was used instead of the exact one. In 
this work, we first present a parallel implementation based on our software stack of the univariate MLOE/MMOM criteria, and then we propose a modified algorithm that extends these criteria to assess the multivariate approximations modeling on large-scale multivariate spatial datasets.

The remainder of the paper is as follows. Section 2 summarizes the contribution of this work. Section 3 recalls some of the established approaches in large-scale multivariate geospatial modeling and inference. Section 4 contains a brief discussion on multivariate geospatial statistics, an introduction to the log-likelihood estimation problem, and a review to some approximation techniques which ameliorate the complexities encountered in MLE operations. Section 5 establishes the research contributions of this paper. Section 6 provides detailed illustrations of our proposed modeling framework on synthetic and real datasets. Section 7 delivers an overall summary and conclusion.

\section{Contributions}

The six-fold contributions of the paper are as follows:

- We present multivariate geospatial modeling and inference in large-scale systems on both exact and TLRbased approximation computations with reduced complexity on the log-likelihood for both estimation and spatial prediction.

- We propose a novel multivariate assessment algorithm based on existing univariate criteria to evaluate our TLR-based parameter estimation accuracy.

- We implement a parallel version of the univariate and the new multivariate criteria to assess the prediction efficiency on synthetic and real datasets.

- We port the proposed implementation on sharedmemory, GPUs, and distributed-memory systems using a modular software stack.

- We evaluate the performance of both the parallel exact and TLR-based MLE computations, as well as the proposed multivariate assessment criteria, using different parallel platforms such as Intel Xeon Skylake/Cascade Lake, AMD EPYC (Rome), ARM ThunderX2, NVIDIA V100 GPU, and a distributedmemory Cray XC40 system.

- We conduct a set of experiments designed to assess the accuracy of our implementation in terms of inference and prediction on both synthetic and real datasets.

\section{RELATED WORK}

Scalable large-scale geospatial statistical modeling has been attempted mostly in the univariate $(p=1)$ setting. The authors in [45], [46], [47], [48], [49] worked on parallelizing the predictions routines using MPI, OpenMP, Parallel Virtual Machines (PVMs) and/or Graphics Processing Units (GPUs). However, their proposals did not include parallelization strategies for computing the inverse of the covariance matrix, which consumes approximately $70 \%$ of the execution time. Nevertheless, they expect a better speedup when the number of locations to be predicted is large.

In parallel kriging literature $(p=1)$, parallel implementations of the Cholesky factorization of the kriging process depend on blocking algorithms and run on a single hardware architecture. For instance, in [50], a parallel implementation of kriging was developed completely on GPU architectures. They proposed an out-of-core GPU implementation of the kriging process using a block-based pivoted Cholesky algorithm which is considered more suitable for GPU compared to CPU. Another example is the parallel framework in [51] which provides end-to-end geospatial analysis from maximum likelihood estimation to kriging using ScaLAPACK to perform distributed-memory implementation on CPUs. However, their framework does not include any GPU implementation. Other strategies focused on replacing the computation of the full covariance matrix with low-rank approximation methods; see Section 4.4 for a review.

When $p>1$, the computations become much more challenging. In light of this, we introduce a framework to deal with large-scale multivariate geospatial statistical modeling that provides both the dense and the TLR-based approximate versions of MLE operations on very large problem sizes. In this work, we depend on the unified software in [42] which is powered by dense linear algebra task-based algorithms and dynamic runtime systems and especially designed for geospatial statistical modeling. The software in [42] also has an equivalent $\mathrm{R}$ implementation described in [52]. The framework we propose depends on the asynchronous task-based dense linear algebra library CHAMELEON [53] and the Hierarchical Computations linear algebra library HICMA [54] for support in dense and lowrank matrix computations, respectively. Both libraries rely on the dynamic runtime system STARPU [55] to exploit the computing power on shared and distributed-memory systems based on multi-core, many-core, and hybrid hardware architectures.

\section{Overview of the Problem}

This section describes multivariate spatial modeling and inference based on a given cross-covariance function, with a brief background on low-rank approximation techniques that have been used in the literature to reduce the complexity of log-likelihood function computations.

\subsection{Cross-Covariance Function}

Quantifying spatial dependence of multiple variables is one of the main foci of multivariate geospatial statistical modeling. The key tool for this purpose is the cross-covariance function. It is a matrix-valued function of dimension $p \times p$, parameterized by $\boldsymbol{\theta} \in \mathbb{R}^{q}$, that describes the degree of dependence between values at two locations $\mathbf{s}_{1}$ and $\mathbf{s}_{2}$, and is of the form $\mathbf{C}(\|\mathbf{h}\| ; \boldsymbol{\theta})=\left\{C_{i j}(\|\mathbf{h}\| ; \boldsymbol{\theta})\right\}_{i, j=1}^{p}$ under the isotropy assumption, where $\mathbf{h}=\mathbf{s}_{1}-\mathbf{s}_{2} \in \mathbb{R}^{d}$, $\|\cdot\|$ denotes the Euclidean norm, and $C_{i j}(\|\mathbf{h}\| ; \boldsymbol{\theta})=$ $\operatorname{cov}\left\{Z_{i}\left(\mathbf{s}_{1}\right), Z_{j}\left(\mathbf{s}_{2}\right)\right\}$. When $i=j, C_{i i}(\|\mathbf{h}\| ; \boldsymbol{\theta})$ is called the marginal covariance function and it measures the spatial dependence between the $i$-th variable at $\mathbf{s}_{1}$ and at $\mathbf{s}_{2}$. When $i \neq j, C_{i j}(\|\mathbf{h}\| ; \boldsymbol{\theta})$ is called the cross-covariance function and it measures the spatial dependence between the $i$-th variable at $\mathbf{s}_{1}$ and the $j$-th variable at $\mathbf{s}_{2}$, for $i, j=1, \ldots, p$. The choice of cross-covariance function $C_{i j}(\|\mathbf{h}\| ; \boldsymbol{\theta})$ is data and 
application-driven. However, $C_{i j}(\|\mathbf{h}\| ; \boldsymbol{\theta})$ needs to ensure that the $\boldsymbol{\Sigma}(\boldsymbol{\theta})$ it builds is a positive definite matrix for any $n \in \mathbb{N}$ and any finite set of points $\mathbf{s}_{1}, \ldots, \mathbf{s}_{n}$.

\subsection{Matérn Cross-Covariance Function}

The Gaussian geospatial statistical modeling landscape is replete with cross-covariance function models. A comprehensive review on the available models can be found in [33. The parsimonious multivariate Matérn is a popular crosscovariance function [56], [57] and has the form

$$
C_{i j}(\|\mathbf{h}\| ; \boldsymbol{\theta})=\frac{\rho_{i j} \sigma_{i i} \sigma_{j j}}{2^{\nu_{i j}-1} \Gamma\left(\nu_{i j}\right)}\left(\frac{\|\mathbf{h}\|}{a}\right)^{\nu_{i j}} \mathcal{K}_{\nu_{i j}}\left(\frac{\|\mathbf{h}\|}{a}\right),
$$

for $i, j=1, \ldots, p$, where $\mathcal{K}_{\nu}(\cdot)$ is the modified Bessel function of the second kind of order $\nu$ and $\Gamma(\cdot)$ is the gamma function. Here $\boldsymbol{\theta}$ includes, for $i=j$, the marginal variance $\left(\sigma_{i i}^{2}>0\right)$, smoothness $\left(\nu_{i i}>0\right)$, and spatial range $(a>0)$ parameters, and for $i \neq j$, the colocated correlation $\left(\rho_{i j}\right)$ and cross smoothness $\left(\nu_{i j}>0\right)$ parameters, such that $\nu_{i j}=\frac{1}{2}\left(\nu_{i i}+\nu_{j j}\right)$ and

$\rho_{i j}=\beta_{i j} \frac{\Gamma\left(\nu_{i i}+\frac{d}{2}\right)^{1 / 2}}{\Gamma\left(\nu_{i i}\right)^{1 / 2}} \frac{\Gamma\left(\nu_{j j}+\frac{d}{2}\right)^{1 / 2}}{\Gamma\left(\nu_{j j}\right)^{1 / 2}} \frac{\Gamma\left\{\frac{1}{2}\left(\nu_{i i}+\nu_{j j}\right)\right\}^{1 / 2}}{\Gamma\left\{\frac{1}{2}\left(\nu_{i i}+\nu_{j j}\right)+\frac{d}{2}\right\}}$,

for any $\sigma_{i i}^{2}, a, \nu_{i i}>0, d \geq 1$. Here $\left(\beta_{i j}\right)_{i, j=1}^{p}$ is a symmetric and positive definite correlation matrix.

The parameter $\rho_{i j}$ describes the dependence or correlation between the $i$-th and $j$-th components situated at the same location, through the latent parameter $\beta_{i j}$. When $\beta_{i j}=0, Z_{i}(\mathbf{s})$ and $Z_{j}(\mathbf{s})$ are independent. Otherwise, when $\beta_{i j}>0\left(\beta_{i j}<0\right)$, the two are positively (negatively) dependent. Sample realizations from the parsimonious bivariate Matérn model above are shown in Fig. 12 where $\boldsymbol{\theta}=\left(\sigma_{11}^{2}, \sigma_{22}^{2}, a, \nu_{11}, \nu_{22}, \beta\right)^{\top}=(1,1,0.2,0.5,1,0.5) \top$.

\subsection{Multivariate Prediction}

The cross-covariance matrix $\boldsymbol{\Sigma}(\boldsymbol{\theta})$ is crucial in obtaining predictions of unknown variables at a prediction location and measuring the uncertainty of these predictions. A prediction location may have all or some variables that are missing. The first case happens when there are locations with sensors that collect measurements for atmospheric variables like temperature, precipitation, and wind speed, for example, and one might be interested in predicting the values of these variables at locations with no sensors. The second case occurs when measurements of one variable are difficult or expensive to obtain while measurements of another variable, correlated with the first one, are easy to collect. In this scenario, there will be more locations with data collection instruments for the cheaper variable, while observations will be sparse for the expensive one. The first case is more prevalent in environmental applications wherein sensors measuring different variables simultaneously were deployed at predetermined sites. Hence, in this work, we assume that all prediction locations are missing the measurements for all $p$ variables. Multivariate geospatial prediction proceeds as follows. Suppose $\mathbf{s}_{0} \in \mathbb{R}^{d}$ is a prediction location with an unknown vector of $p$ variables $\mathbf{Z}\left(\mathbf{s}_{0}\right)$. Under the squarederror loss criterion, the best linear unbiased predictor of
$\mathbf{Z}\left(\mathbf{s}_{0}\right)$, given $\mathbf{Z}=\left\{\mathbf{Z}\left(\mathbf{s}_{1}\right)^{\top}, \ldots, \mathbf{Z}\left(\mathbf{s}_{n}\right)^{\top}\right\}^{\top}$, also called the cokriging predictor, is

$$
\widehat{\mathbf{Z}}\left(\mathbf{s}_{0}\right)=\mathbf{c}_{0}^{\top} \boldsymbol{\Sigma}(\boldsymbol{\theta})^{-1} \mathbf{Z} .
$$

Here $\mathbf{c}_{0}$ is the $p n \times p$ matrix formed by taking the crosscovariance between $\mathbf{Z}\left(\mathbf{s}_{0}\right)$ and $\mathbf{Z}\left(\mathbf{s}_{r}\right)$, at all sampled locations $\mathbf{s}_{r}, r=1, \ldots, n$, i.e.,

$$
\mathbf{c}_{0}=\left\{\mathbf{C}\left(\mathbf{s}_{0}-\mathbf{s}_{1} ; \boldsymbol{\theta}\right), \ldots, \mathbf{C}\left(\mathbf{s}_{0}-\mathbf{s}_{n} ; \boldsymbol{\theta}\right)\right\}^{\top} .
$$

\subsection{Low-Rank Approximation}

Gaussian geospatial statistical modeling relies heavily on the operations done on $\boldsymbol{\Sigma}(\boldsymbol{\theta})$. In the early stages of modeling, $\boldsymbol{\Sigma}(\boldsymbol{\theta})$ has to be formed by evaluating the crosscovariance function at $n$ locations, for all $p$ variables. Parameters then have to be estimated, with the cross-covariance function evaluated every time new sets of parameters are assumed. Further, the Gaussian log-likelihood in Equation (1) requires the inverse and the determinant of $\boldsymbol{\Sigma}(\boldsymbol{\theta})$. Prediction also involves the inverse of $\boldsymbol{\Sigma}(\boldsymbol{\theta})$.

Several techniques to bypass these computing obstacles by exploiting data sparsity have been proposed. Low-rank approximations have been widely used for data modeling. Several low-rank representations of the original Gaussian processes had been proposed during recent years, including predictive process [58], where a select set of knots is used to approximate the original process and a low-rank model is obtained. Later, the modeling approaches were extended to multi-resolution approximation [59] to capture spatial structures from different scales. A low-rank approximation can also be applied to Vecchia's representation for the composite likelihood |60|, resulting in reduced computational burden in obtaining the composite likelihood. Moreover, the lowrank approximation can also be applied to cross-covariance matrices with limited loss of information. Another possibility to facilitate computations for exascale modeling is to reduce the complexity of $\boldsymbol{\Sigma}(\boldsymbol{\theta})$ directly. Covariance tapering [61], for example, forces $\boldsymbol{\Sigma}(\boldsymbol{\theta})$ to be sparse by introducing a compact support. This technique is also known as the Diagonal Super Tile (DST) wherein the entries of the tiles that are very far from the diagonal are annihilated or reduced to zero [3]. Bayesian hierarchical models have also been championed in large-scale geospatial statistical analyses |62], |63|.

\subsection{Univariate MLOE/MMOM}

A common metric used to assess the quality of the predictions is the MSPE and is computed as MSPE = $\frac{1}{n_{\text {pred }}} \sum_{l=1}^{n_{\text {pred }}}\left\|\widehat{\mathbf{Z}}\left(\mathbf{s}_{0, l}\right)-\mathbf{Z}\left(\mathbf{s}_{0, l}\right)\right\|^{2}$, where $\mathbf{s}_{0,1}, \mathbf{s}_{0,2}, \ldots, \mathbf{s}_{0, n_{\text {pred }}}$ are the $n_{\text {pred }}$ prediction locations. When the predictions are obtained through an approximated cross-covariance matrix, e.g., the TLR version of the exact covariance matrix, an appropriate metric should be used. The authors in |44 suggested the use of the MLOE and MMOM. However, their formulations are only available for univariate predictions $(p=1)$. The formulas and the algorithm for the univariate MLOE/MMOM can be found in [44]. In Section 5.4, we extend these metrics and the algorithm to multivariate. 


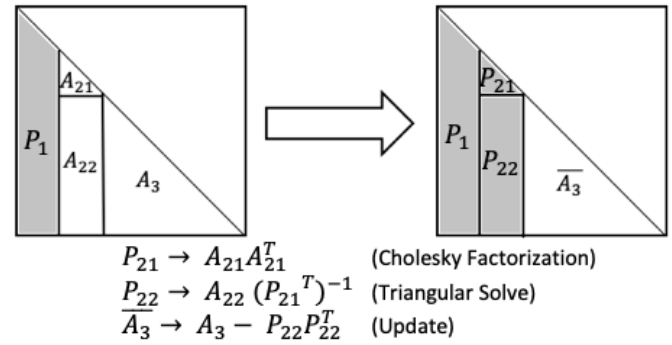

Fig. 1: One Shot of the Block Cholesky Factorization Algorithm.

\section{Proposed Framework}

In this section, we explain in detail our software dependencies, proposed multivariate modeling and inference implementation, and multivariate MLOE/MMOM criteria which are used to assess the accuracy of the multivariate modeling.

\subsection{Parallel Software Architecture}

Our proposed framework internally relies on a list of software dependencies including CHAMELEON [53], STARPU [55], HICMA [54], STARS-H [64], and NLOPT [65], as demonstrated in Fig. 2 CHAMELEON is a tile-based high-performance numerical library based on task-parallel programming models which offer a more structured way of expressing parallelism using three backend runtime systems, namely, QUARK [66], PARSEC [67], and STARPU.

In the literature, parallel linear algebra operations were performed in parallel systems using block-column algorithms. These algorithms disband the given matrix, represented in column-major format, into a successive panel and update the computational phases. Assuming that Cholesky factorization is performed, the factorization is applied to each panel, and the matrix transformations are blocked and applied together at one time during the update phase; see Fig. 1. LAPACK and ScaLAPACK are examples of dense linear algebra library on shared-memory and distributedmemory systems, respectively. CHAMELEON adopts tile algorithms methodology by splitting the given matrix into a set of tiles, instead of panels, which allows updating the trailing submatrix before factorization is complete. These algorithms aim at weakening the synchronization points while performing matrix operations and maximization of the utilization of underlying hardware resources. The numerical algorithm can then be translated into a Directed Acyclic Graph (DAG), where the nodes represent tasks and the edges define data dependencies (e.g., read, write, and read-write), as shown in Fig. 2 with a $4 \times 4$ Cholesky factorization DAG example. Runtime systems lead the rudder of utilizing the usage of underlying hardware resources in CHAMELEON, allowing tile algorithms to run efficiently on different parallel hardware with homogeneous and heterogeneous architectures. DAG tasks are scheduled across different hardware resources to ensure that the data dependencies rules predefined by the user are not violated. Runtime systems enhance software productivity by abstracting the hardware complexity from the end-user. They are also capable of mitigating data movement overhead, reducing load imbalance, and increasing hardware utilization.

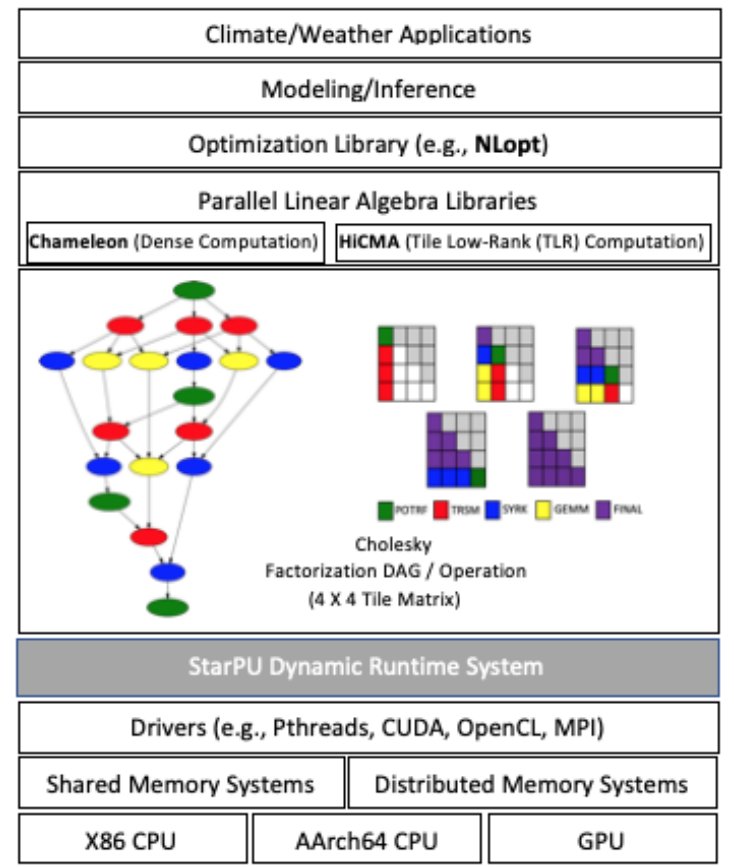

Fig. 2: Software architecture based on NLOPT, CHAMELEON, HICMA, and STARPU for climate/weather applications with a $4 \times 4$ Cholesky factorization DAG example.

Here, we use the STARPU dynamic runtime system because of its ability to support a wide range of parallel heterogeneous hardware architectures from different vendors like Intel, AMD, NVIDIA, and ARM. STARPU executes defined generic task graphs, generated by a built-in sequential task flow (STF) programming model. STARPU scheduler pushes the set of tasks to the available processing unit based on these dependencies which may lead to asynchronous execution. STARPU supports different programming languages (e.g., Pthreads, CUDA, OpenCL, and MPI) and runs on different hardware architectures (e.g., CPU/GPU, shared/distributed-memory). STARPU may decide at runtime to execute the tasks on different hardware based on performance models.

HICMA supports parallel TLR matrix computation. It relies on CHAMELEON with STARPU as the runtime and STARS-H as the compressed matrix generator. NLOPT is an open-source $\mathrm{C} / \mathrm{C}++$ nonlinear optimization toolbox which we rely on to perform the optimization task for the MLE operation. STARPU runtime system handles both exact dense and TLR computational workloads to perform the required linear algebra operations in parallel.

\subsection{Exact Multivariate Modeling}

In multivariate modeling, there are two ways to build $\boldsymbol{\Sigma}(\boldsymbol{\theta})$ [33]. The first approach (Representation I) is to build an $n \times n$ matrix with block elements of $p \times p$ matrices $\mathbf{C}\left(\mathbf{s}_{l}-\mathbf{s}_{r} ; \boldsymbol{\theta}\right), l, r=1, \ldots, n$. The second approach (Representation II) is to build a $p \times p$ matrix with block elements of $n \times n$ matrices $\left\{C_{i j}\left(\mathbf{s}_{l}-\mathbf{s}_{r} ; \boldsymbol{\theta}\right)\right\}_{l, r=1}^{n}, i, j=1, \ldots, p$. To illustrate, suppose $p=2$ and $n=3$. Fig. 3 shows $\boldsymbol{\Sigma}(\boldsymbol{\theta})$ of dimension $6 \times 6$ under the two representations. 

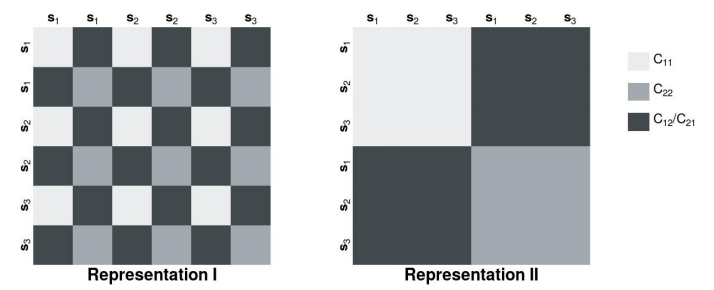

Fig. 3: The order of the elements of $\boldsymbol{\Sigma}(\boldsymbol{\theta})$ is drawn under the two different cross-covariance matrix representations.

The two representations in Fig. 3 yield a symmetric positive definite matrix. Cholesky factorization, the backbone of MLE, is performed on this symmetric positive definite matrix to obtain its inverse and log determinant required for maximizing the log-likelihood function in Equation (1).

A simulation study on the parsimonious bivariate Matérn was conducted to assess the efficiency in parameter estimation and accuracy of predictions under the two representations via comparison of the medians and standard deviations of the estimated parameters and the MSPE of the predictions. The results indicate that the two representations are numerically equivalent in exact computation and either one can substitute for the other. Thus, only Representation I of the exact multivariate model is utilized in this work.

To hasten parameter estimation of the exact parsimonious Matérn cross-covariance function, we maximize the profile log-likelihood in lieu of the full log-likelihood in Equation (1). The profile likelihood is a variant of the full log-likelihood wherein the number of parameters to be estimated is effectively reduced [68]. Under this approach, the marginal variance parameters $\sigma_{i i}^{2}$, for $i=1, \ldots, p$, are no longer included in the estimation and can be derived after all the other parameters were estimated, i.e., $\hat{\sigma}_{i i}^{2}=n^{-1}\left\{\mathbf{Z}_{i}^{\top} \mathbf{R}_{i i}\left(\hat{\boldsymbol{\theta}}_{i}\right)^{-1} \mathbf{Z}_{i}\right\}$, where $\hat{\boldsymbol{\theta}}_{i} \in \mathbb{R}^{q_{i}}, q_{i} \geq 1$, is the set of $q_{i}$ estimated marginal parameters for $Z_{i}$, except the marginal variance parameter $\sigma_{i i}^{2}$, i.e., $\hat{\nu}_{i i}, \hat{a}$, and $\mathbf{R}_{i i}\left(\hat{\boldsymbol{\theta}}_{i}\right)$ is the correlation matrix formed by evaluating the crosscovariance function for $i=j$, using $\hat{\boldsymbol{\theta}}_{i}$ and $\sigma_{i i}^{2}=1$, for $i=1, \ldots, p$. Here $\mathbf{Z}_{i}$ is the vector formed by all the values pertaining to variable $i$, i.e., $\mathbf{Z}_{i}=\left\{Z_{i}\left(\mathbf{s}_{1}\right), \ldots, Z_{i}\left(\mathbf{s}_{n}\right)\right\}^{\top}$.

\subsection{TLR-Based Multivariate Modeling}

Through the last decade, tile algorithms were created to adapt to parallel architectures that require data sharing [69]. The tiling mechanism improved block-based algorithm which suffers from the existence of numerous synchronization points that slow down the overall performance.

TLR approximation algorithms have been implemented based on the tiling technique. Instead of applying the lowrank approximation to the whole matrix, each tile is compressed as a separate unit. Here, we rely on the TLR implementation of [70], where the authors have implemented the TLR approximation by performing the singular value decomposition (SVD) algorithm for each off-diagonal tile by preserving the most significant values and vectors in the corresponding tile, i.e., the tile rank. The diagonal tiles are kept dense since they cannot be approximated. Ranks of

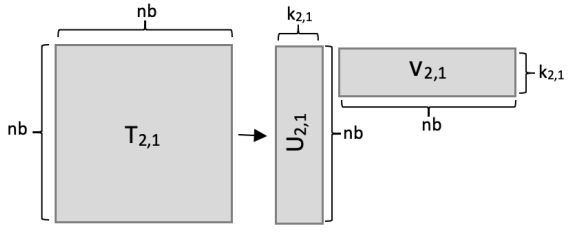

Fig. 4: An example of TLR approximation tile: $T_{2,1}$ with dimension $n b \times n b$ is approximated by two matrices $U_{2,1}$ and $V_{2,1}$ with dimension $n b \times k_{2,1}$
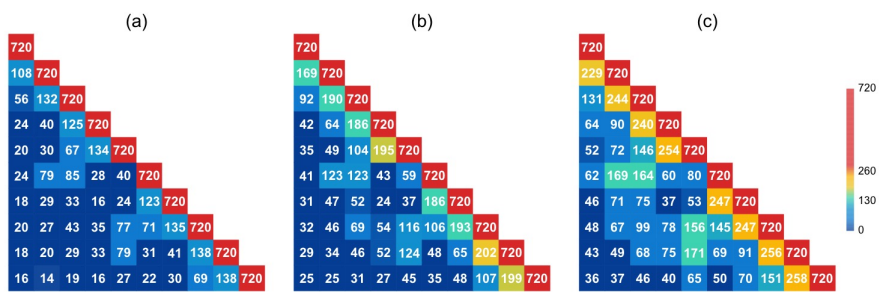

Fig. 5: Rank distributions of a $7200 \times 7200$ bivariate covariance matrix using $n b=720$ with parsimonious bivariate Matérn parameters $\boldsymbol{\theta}=(1,1,0.09,0.5,1,0.5)^{\top}$ under (a) TLR5, (b) TLR7, and (c) TLR9.

the off-diagonal tiles are determined based on the accuracy requirement of the application. Fig. 4 shows an example of compressing an off-diagonal tile $T_{2,1}$ to two matrices $U_{2,1}$ and $V_{2,1}$.

The effectiveness of the TLR mechanism depends on the ranks of the matrix tiles, which in turn depend on the accuracy requirements of the given application. To reduce the ranks of the covariance matrix tiles, we ordered the matrix based on the Morton ordering approach |71], which matches with Representation I in Fig. 3 To validate the usage of the TLR approximation with multivariate modeling, we estimate the ranks of the generated covariance matrix tiles with different accuracy levels, namely, TLR5 $\left(10^{-5}\right)$, TLR7 $\left(10^{-7}\right)$, and TLR9 $\left(10^{-9}\right)$, on a $7200 \times 7200$ bivariate covariance matrix; see Fig. 5 The ranks distribution shows that the off-diagonal ranks grow as the tiles get closer to the diagonal. It can also be observed that even with a higher accuracy, e.g., TLR9, the ranks are still small compared to the ranks of the full dense tiles in the diagonal (in red). The example was drawn from a synthetic set of parameters $\boldsymbol{\theta}=\left(\sigma_{11}^{2}, \sigma_{22}^{2}, a, \nu_{11}, \nu_{22}, \beta\right)^{\top}=(1,1,0.09,0.5,1,0.5)^{\top}$, which represents a moderate spatial dependence between two variables, $Z_{1}$ and $Z_{2}$. Other sets of parameters representing varying strengths of spatial dependence were also examined and the results do not differ significantly from what is shown in Fig. 5 .

Fig. 6 shows the memory footprint of the requirement of full MLE operation for dense, TLR5, TLR7, and TLR9 on various multivariate problem sizes. The memory footprint involves $\boldsymbol{\Sigma}(\boldsymbol{\theta})$ of dimension $2 n \times 2 n$ and two measurement vectors, $Z_{1}$ and $Z_{2}$. The measurement vectors are always represented in the exact format since there is no benefit in compressing them. As shown, the TLR-based compression requires less memory footprint with respect to the dense representation. On average, the TLR representations require $6.68 \mathrm{X}, 4.93 \mathrm{X}$, and $3.86 \mathrm{X}$ less memory than the dense 


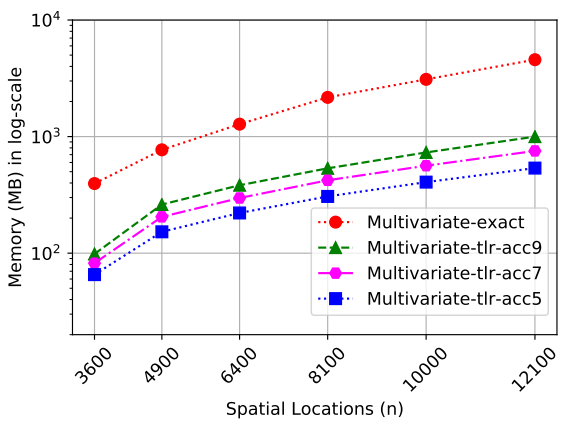

Fig. 6: Memory footprint of exact and TLR-based MLE with varying size of $n$ and two measurement vectors $Z_{1}$ and $Z_{2}$.

representation for $10^{-5}, 10^{-7}$, and $10^{-9}$, respectively. The memory saving increases with larger problem sizes, as seen in the figure.

The performance model of TLR is driven by the most time-consuming kernel, i.e., the TLR matrix-matrix multiplication (TLR-MM). The arithmetic complexity of a single TLR-MM is $36 \times n b \times k^{2}$ [72], with $n b$ the tile size and $k$ the tile rank that depends on the number of significant singular values after compression. The total number of operations is $O\left(n^{2} k\right)$, attained when $n b=O(\sqrt{n})$. This tile size is a trade-off between the arithmetic intensity of the kernel and the degree of parallelism of the algorithm. The performance model of TLR is driven by a quadratic regime, which contrasts with the cubic regime for exact computations. The detailed complexity analysis of TLR Cholesky factorization can be found in [73]. We are also looking into more advanced matrix compression strategies [74], [75], [76] that exhibit better arithmetic complexity but these may be challenging to implement on massively parallel systems due to their hierarchical structures.

\subsection{Proposed Multivariate MLOE/MMOM Criteria}

Assessing the estimation accuracy of the modeling approach is challenging and requires a well-developed algorithm. Our novel multivariate prediction assessment metrics depend on the MLOE/MMOM [44]. There are two possible multivariate versions of the MLOE/MMOM. The first version is a naive extension and it is simply the mean of the MLOE/MMOMs of all the variables. This approach requires univariate covariance models and the univariate version of the cokriging equation (3). Another version, which we propose, is to utilize cross-covariance functions and the cokriging equation (3). Denote the cokriging error vector by $\mathbf{e}\left(\mathbf{s}_{0}\right)=\left(e_{1}, \ldots, e_{p}\right)^{\top}$, where $e_{i}=\hat{Z}_{i}\left(\mathbf{s}_{0}\right)-Z_{i}\left(\mathbf{s}_{0}\right)$ and $\hat{Z}_{i}\left(\mathbf{s}_{0}\right)$ is the predictor for variable $i, i=1, \ldots, p$, at a prediction location $\mathbf{s}_{0}$, obtained using the cokriging equation (3), with the true cross-covariance function parameters, $\boldsymbol{\theta}$. The mean square error of this predictor is

$$
\mathrm{E}_{t}=\operatorname{tr}\left\{\mathbf{C}(\mathbf{0} ; \boldsymbol{\theta})-\mathbf{c}_{0}^{t^{\top}} \boldsymbol{\Sigma}(\boldsymbol{\theta})^{-1} \mathbf{c}_{0}^{t}\right\},
$$

where the subscript $t$ in $\mathbf{c}_{0}^{t}$ specifies that the parameters used in Equation (4) are the true parameters $\boldsymbol{\theta}$ and tr indicates the trace of the matrix. Suppose now that the set of estimated parameters derived from using a certain approximation of the covariance matrix, $\hat{\boldsymbol{\theta}}^{a}$, were used to build the cokriging equation (3). The error introduced by the approximation is $\mathbf{e}_{a}\left(\mathbf{s}_{0}\right)=\left(e_{a, 1}, \ldots, e_{a, p}\right)^{\top}$, where $e_{a, i}=\hat{Z}_{i}^{a}\left(\mathbf{s}_{0}\right)-Z_{i}\left(\mathbf{s}_{0}\right)$ and $\hat{Z}_{i}^{a}\left(\mathbf{s}_{0}\right)$ is the predictor for variable $i$ at a prediction location $\mathbf{s}_{0}$, obtained using the cokriging equation in $(3)$ with $\hat{\boldsymbol{\theta}}^{a}$. The mean square error of this predictor is

$$
\begin{aligned}
\mathrm{E}_{t, a}=\operatorname{tr}\{\mathbf{C}(\mathbf{0} ; \boldsymbol{\theta})- & 2 \mathbf{c}_{0}^{t^{\top}} \boldsymbol{\Sigma}\left(\hat{\boldsymbol{\theta}}^{a}\right)^{-1} \mathbf{c}_{0}^{a} \\
& \left.+\mathbf{c}_{0}^{a^{\top}} \boldsymbol{\Sigma}\left(\hat{\boldsymbol{\theta}}^{a}\right)^{-1} \boldsymbol{\Sigma}(\boldsymbol{\theta}) \boldsymbol{\Sigma}\left(\hat{\boldsymbol{\theta}}^{a}\right)^{-1} \mathbf{c}_{0}^{a}\right\}
\end{aligned}
$$

where $\mathbf{c}_{0}^{a}$ is Equation (4) evaluated using $\hat{\boldsymbol{\theta}}^{a}$ and $\boldsymbol{\Sigma}\left(\hat{\boldsymbol{\theta}}^{a}\right)$ is the cross-covariance matrix also evaluated using $\hat{\boldsymbol{\theta}}^{a}$. The subscript $t, a$ in $\mathrm{E}_{t, a}$ specifies that given the true parameters $\boldsymbol{\theta}$, the estimated parameters $\hat{\boldsymbol{\theta}}^{a}$ from the approximated model are used instead.

The multivariate MLOE/MMOM, denoted as $\mathrm{MLOE}^{C K}$ and $\mathrm{MLOE}^{C K}$, respectively, are as follows:

$$
\begin{aligned}
\operatorname{MLOE}^{C K} & =\frac{1}{n_{\text {pred }}} \sum_{l=1}^{n_{\text {pred }}} \operatorname{LOE}^{C K}\left(\mathbf{s}_{0, l}\right) \\
\mathrm{MMOM}^{C K} & =\frac{1}{n_{\text {pred }}} \sum_{l=1}^{n_{\text {pred }}} \operatorname{MOM}^{C K}\left(\mathbf{s}_{0, l}\right),
\end{aligned}
$$

where $\operatorname{LOE}^{C K}\left(\mathbf{s}_{0}\right)=\frac{\mathrm{E}_{t, a}}{\mathrm{E}_{t}}-1$ and $\operatorname{MOM}^{C K}\left(\mathbf{s}_{0}\right)=\frac{\mathrm{E}_{a}}{\mathrm{E}_{t, a}}-1$. The superscript $C K$ stipulates that the multivariate extensions were derived from the cokriging equation (3) and $\mathrm{E}_{a}$ in Equation (5) evaluated using $\hat{\boldsymbol{\theta}}^{a}$ and $\mathbf{c}_{0}^{a}$.

The algorithm implementing this approach for $p=$ 2 is outlined in Algorithm 11. This new algorithm is similar to |44 except now the matrix-valued Matérn cross-covariance function is utilized instead of the scalar-valued univariate Matérn covariance function, e.g., $\operatorname{BiMatérn}\left(\mathbf{s}_{1}, \mathbf{s}_{2} ; \boldsymbol{\theta}\right)$ returns a $2 \times 2$ matrix.

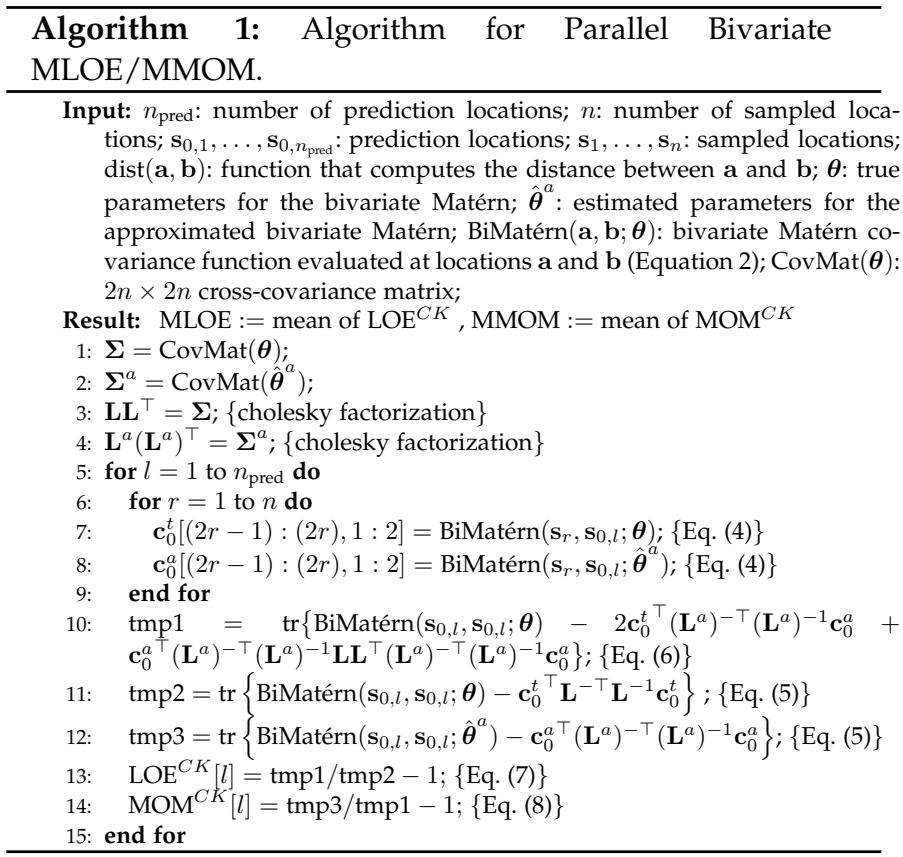

Assuming $n_{\text {pred }} \ll n$, the memory footprint and the arithmetic complexity of Algorithm 1 for any values of $p$ 
depends solely on the Cholesky factorizations of $\boldsymbol{\Sigma}$ and $\boldsymbol{\Sigma}^{a}$ (lines 3 and 4). Each Cholesky factorization requires $p^{2} n^{2}$ memory footprint and $(1 / 3) p^{3} n^{3}$ number of operations. Indeed, the code section containing the nested loops (lines 515) carries only Level- 1 and Level-2 BLAS operations involving several dot products and triangular solves. Since these matrix operations account for the lower order terms [77], the overall memory footprint then is $2 p^{2} n^{2}$ and the arithmetic complexity is $(2 / 3) p^{3} n^{3}$.

\section{Performance Results}

In this section, we assess the performance and accuracy of the TLR approximation to $\boldsymbol{\Sigma}(\boldsymbol{\theta})$ on large-scale simulations and real datasets. The performance assessment involves a wide range of parallel hardware systems while the accuracy assessment entails simulating synthetic datasets from the parsimonious Matérn cross-covariance function, estimating the model parameters, and predicting values at screened locations. Estimation and prediction accuracy are also assessed on real datasets. The designed experiments show that the TLR approximation outperforms the exact bivariate computation while maintaining the accuracy required by geospatial statistics applications.

\subsection{Testbed and Methodology}

All experiments described in this paper were performed using a variety of shared-memory systems, including a dualsocket 28-core Intel Skylake Intel Xeon Platinum 8,176 CPU running at $2.10 \mathrm{GHz}$, a dual-socket 20-core Intel Cascade Lake Intel Xeon Gold 6,248 CPU running at $2.50 \mathrm{GHz}$, a dual-socket 64-core AMD EPYC (Rome) 7702, a dual-socket 20-core Intel Skylake/V100 GPU Intel Xeon Gold 6,148 CPU running at $2.40 \mathrm{GHz}$, and 32-core ARM ThunderX2 Cavium at $2.10 \mathrm{GHz}$. For the distributed-memory experiments, we use a Cray XC40 system, with 6,174 16-core Intel dual-socket Haswell processors running at $2.3 \mathrm{GHz}$, where each node has 128 GB of DDR4 memory. The KAUST Shaheen-II Cray XC40 system has a total of 197,568 processor cores and 790 $\mathrm{TB}$ of aggregate memory.

To obtain timing results, we run each simulation three times on every single hardware with the same configuration and report the average. We find runtime variations between $0.1 \%$ and $0.5 \%$ on shared-memory systems and between $1 \%$ and $3 \%$ on distributed-memory system. The latter is slightly higher since the runs are subject to network fluctuations depending on the current load of the system.

\subsection{TLR-Based Bivariate MLE Performance}

In this section, we evaluate the TLR-based multivariate MLE performance and compare it to the performance of the exact MLE. All the experiments show one iteration of the MLE optimization since all the iterations have the same complexity for both exact and TLR-based computation. Fig.7 7 shows the TLR performance on different shared-hardware architectures. The execution time is shown in the Y-axis (identical for all hardware) while the number of spatial locations is shown in the X-axis. We use TLR5 as the benchmark of the speedup gained by the TLR computation.
With an Intel 56-core Skylake system, TLR5 can achieve on average $4 \mathrm{X}$ speedup compared to exact MLE, while on an Intel 40-core Cascade Lake system, the average speedup can reach 4.3X. With a 128-core AMD EPYC (Rome) system and a 64-core ThunderX2 ARM system, the average speedup reaches to $6 \mathrm{X}$ and 5.5X, respectively. All figures reveal more gains from the TLR-based approximation with larger problem sizes. Moreover, with a larger number of cores, the average speedup factor achieved increases.

To compare the gained speedup from each hardware architecture in Fig. 77 we use the same number of locations $n=63,001$ as a reference. The execution time of one full bivariate TLR-based MLE iteration (TLR5) is 61.38, 65.65, 35.75, and 113.21 seconds on Intel Skylake, Intel Cascade lake, AMD EPYC (Rome), and ThunderX2 ARM chips, respectively. From the speedup perspective, AMD EPYC chip achieves the best performance compared to the other systems. It obtains 1.7X, 1.8X, and 3.17X speedup compared to Intel Skylake, Intel Cascade lake, and ThunderX2 ARM systems.

The target hardware systems have different number of cores which makes comparing them more difficult. Given that the implementation is memory-bound, we rely on the sustained bandwidth on each system to give more insights into the obtained performance. Intel Skylake, Intel Cascade lake, AMD EPYC (Rome), and ThunderX2 ARM have a sustained bandwidth (measured by STREAM benchmark [78|) 178, 140, 300, $236 \mathrm{~GB} / \mathrm{s}$. Based on these memory bandwidth values, we can expect that AMD EPYC (Rome) satisfies $300 / 178=1.68 \mathrm{X}$ speedup compared to Intel Skylake, $300 / 140=2.14 \mathrm{X}$ compared to Intel Cascade Lake, and 1.3X compared to ThunderX2 ARM. The calculations show close values to the obtained speedup except for the ThunderX2 ARM chip. The speedup discrepancy shows that more parallelism needs to be exposed to the ThunderX2 ARM chip to take more advantage of its 64 cores.

On the Cray XC40 distributed-memory system, TLR achieves lower speedup compared to the exact but still outperforms it with different problem size and number of nodes. Tuning the tile size $(n b)$ is challenging on distributedmemory systems and it seems that our baseline runtime systems, i.e., STARPU, impacts performance with a large number of nodes. Fig. Ba shows the performance of different TLR accuracy with problem size up to $325 \mathrm{~K}$ on 64 nodes. The average speedup gained is about $2 \mathrm{X}$. With 128 nodes, the average speedup gained is about $1.8 \mathrm{X}$ as shown by Fig. $8 \mathrm{~b}$

Fig. 9 shows the strong scalability results using single node 40-core Intel Cascade Lake system with different number of cores and Cray XC40 machine using different number of nodes (up to 128 nodes). In Fig. 9a, the Cascade Lake system shows decent parallel speedup as we increase the numbers of threads with $n=63,001$. The parallel efficiency on average is around $72 \%$, (i.e., $T_{\min } /\left(N * T_{N}\right) * 100 \%$, where one thread execution time is $T_{1}$ and $N$ threads execution time is $T_{N}$ ), compared to single thread executions and across different computation variants, i.e., exact, TLR5, TLR7, and TLR9. In Fig. 9b, the exact computation achieves around 66.7X speedup, while the TLR approximation with different accuracy levels obtains around 51.7X speedup on average with different number of nodes using $n=168,100$. The 


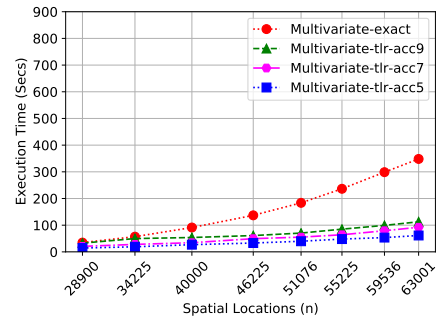

(a) 56-core Intel Skylake

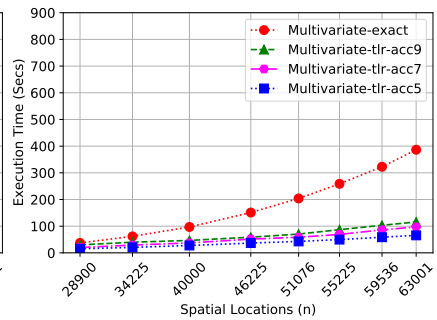

(b) 40-core Intel Cascade Lake

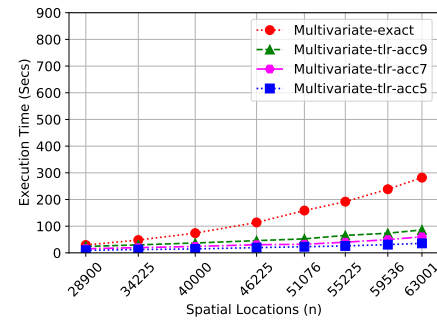

(c) 128-core AMD EPYC

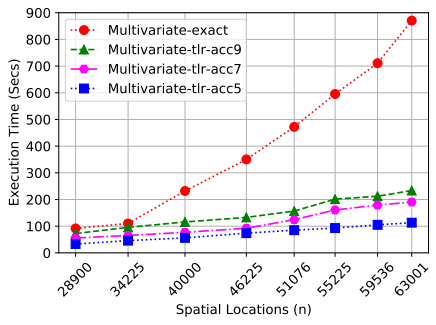

(d) 64-core ThunderX2 ARM

Fig. 7: One bivariate exact and TLR-based MLE iteration using different matrix sizes on various shared-memory systems.
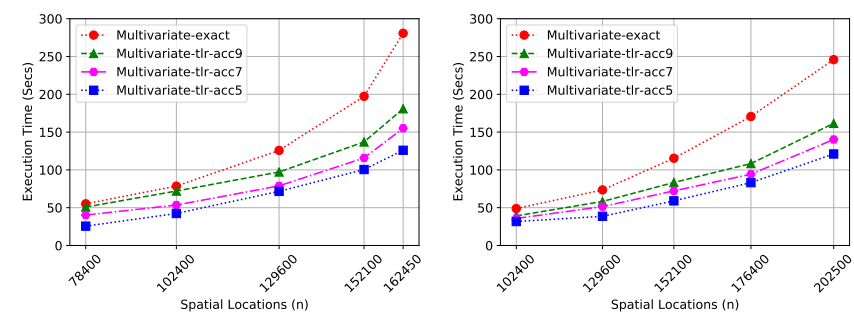

(a) Cray XC40 - 64 nodes

Fig. 8: One bivariate exact and TLR-based MLE iteration using different matrix sizes on various shared-memory systems.
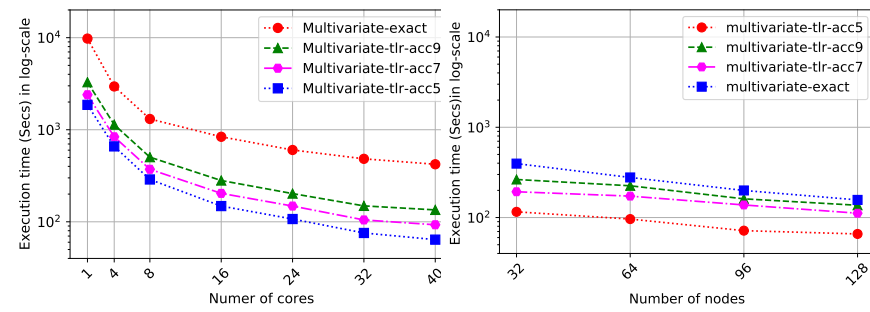

(a) 40-core Intel Cascade Lake (b) Cray XC40 with different with different number of number of nodes. cores.

Fig. 9: Strong scalability plot of one bivariate exact and TLRbased MLE iteration.

parallel efficiency of the TLR approximation varies between $60 \%$ and $43 \%$. This is lower than the efficiency of the exact computation but expected due to the memory-bound versus compute-bound regime of executions opposing TLR and exact computations, respectively.

\subsection{Univariate/Bivariate MLOE/MMOM Criteria Perfor- mance}

In this set of experiments, we aim at assessing the performance of the proposed multivariate MLOE/MMOM criteria algorithm. We choose the bivariate as an example of a multivariate case with synthetic datasets generated by our framework. The experiments were performed on different shared-memory hardware architectures. We provide the time breakdown of the assessment operation of Algorithm 1 and split them into three parts: matrices generation time (GEN_TIME) in lines 1-2, factorization time (FACT_TIME) in lines 3-4, and computation time

(COMP_TIME) in lines 5-15. Figs. 10 and 11 show the time for each operation on both parallel univariate and bivariate MLOE/MMOM implementations.

As shown in both figures, the COMP_TIME is the most time-consuming part of Algorithm 1 as it requires an iterative execution loop equal to the number of missing locations, i.e., 100 in this set of experiments. However, with a larger matrix size, the FACT_TIME takes more time for the whole operation. One striking observation from Fig. 10d is that using a system with V100 GPU speeds up the FACT_TIME compared to COMP_TIME. This is because the computation part involves several matrixvector operations (i.e., Level-2 BLAS) that cannot exploit the computational power of the enclosed GPU.

\subsection{TLR-Based Bivariate MLE Accuracy Assessment}

Here, we assess the accuracy of the proposed TLR-based bivariate MLE using synthetic datasets.

\subsubsection{Synthetic Datasets}

We perform large-scale simulations from the parsimonious Matérn cross-covariance function. Fig. 12 shows a bivariate random field simulated from Equation (2) at $n=24,964$ locations using our synthetic data generator with the following configuration:

- $\beta=0.5$, i.e., $Z_{1}$ and $Z_{2}$ are positively correlated. This parameter controls how correlated $Z_{1}$ and $Z_{2}$ at any location. The effect of this parameter is visually detectable since wherever there are red (blue) spots in $Z_{1}$, red (blue) spots in $Z_{2}$ tend to also be seen.

- $\nu_{11}=0.5$ and $\nu_{22}=1$, i.e., $Z_{2}$ is smoother than $Z_{1}$. The smoothness parameters show through observing that the values of $Z_{2}$ changes more slowly than the values of $Z_{1}$ from one pixel or location to another.

- $\quad a=0.2$. This parameter affects $Z_{1}$ and $Z_{2}$ in different ways. For $Z_{1}$, this value of the scale parameter suggests that the marginal covariance of $Z_{1}$ drops to 0.05 when the locations are 0.6 units apart. For $Z_{2}$, it takes 0.8 units separation for its marginal covariance to drop to 0.05 . Visually, this parameter dictates the sizes of the red and blue spots. The larger the $a$ becomes, the bigger the sizes of the spots are.

To validate the accuracy of the TLR approximation, we simulate 50 different bivariate Gaussian random fields of the same configuration as the example in Fig. 12 and perform three kinds of experiments: 


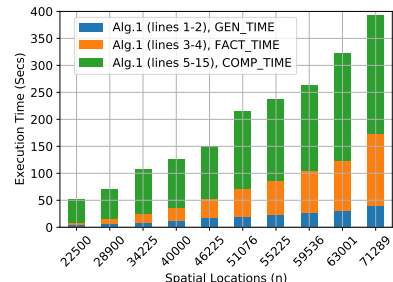

(a) 56-core Intel Skylake

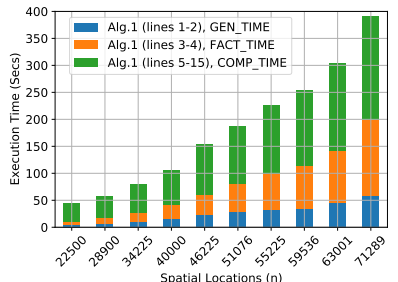

(b) 40-core Intel Cascade Lake

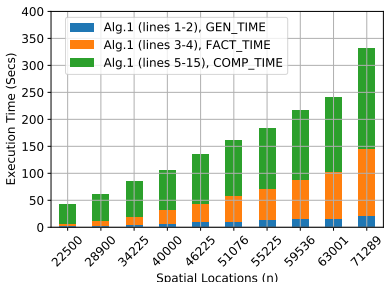

(c) 128-core AMD EPYC

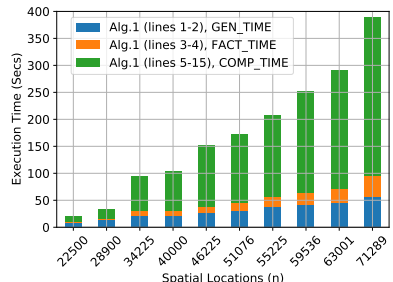

(d) 40-core Intel Skylake +V100

Fig. 10: Time breakdown of the univariate MLOE/MMOM criteria using 100 missing locations on different shared-memory systems.

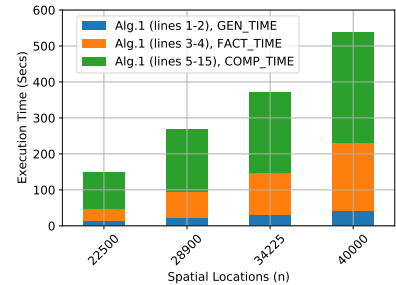

(a) 56-core Intel Skylake

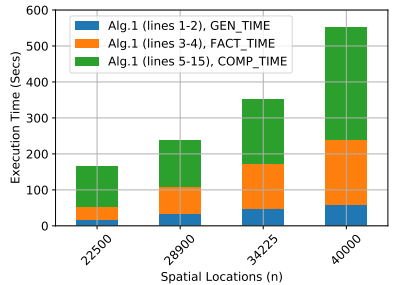

(b) 40-core Intel Cascade Lake

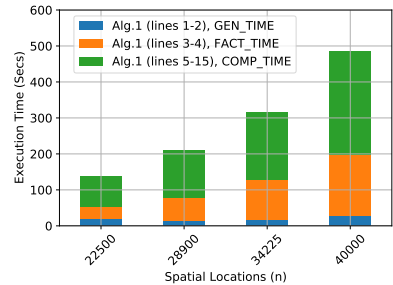

(c) 128-core AMD EPYC

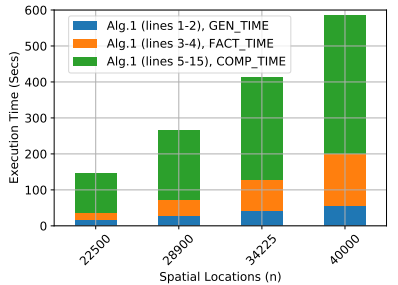

(d) 40-core Intel Skylake +V100

Fig. 11: Time breakdown of the bivariate MLOE/MMOM criteria using 100 missing locations on different shared-memory systems.
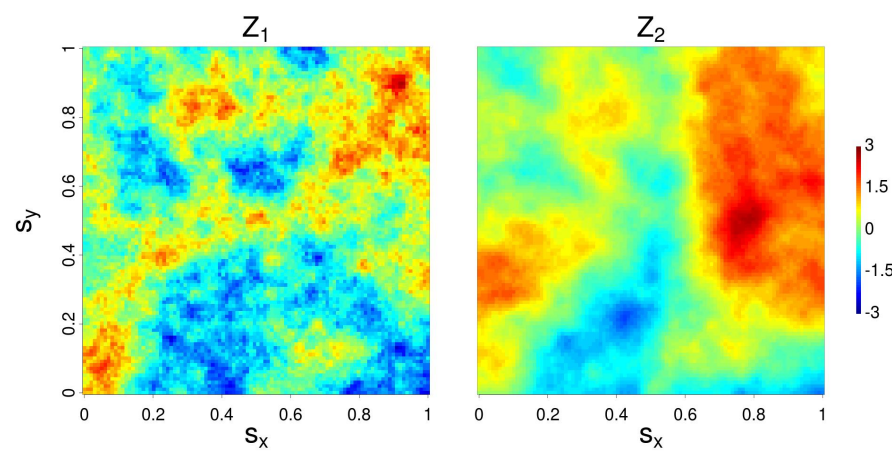

Fig. 12: Spatial images of the simulated bivariate realizations from the parsimonious Matérn cross-covariance model on a $158 \times 158$ regular grid on a unit square. Here $\sigma_{11}^{2}=\sigma_{22}^{2}=1$, $a=0.2, \nu_{11}=0.5, \nu_{22}=1$, and $\beta=0.5$.

- Experiment 1: We show the merits of bivariate spatial modeling in the exact computations by varying the degree of colocated dependence between $Z_{1}$ and $Z_{2}$, controlled by $\rho$ through $\beta$, and examining whether there is a gain in prediction when using the parsimonious bivariate Matérn for different values of $\beta$, while fixing the other parameters as follows. $\sigma_{11}^{2}=\sigma_{22}^{2}=1, \nu_{11}=0.5, \nu_{22}=1$, and $a=0.09$.

- Experiment 2: We examine the quality of parameter estimates for the exact and TLR under different accuracies (TLR5, TLR7, and TLR9), using the available data in $n=22,464$ locations (chosen randomly) and reserving the remaining $n_{\text {pred }}=2,500$ as prediction locations. Furthermore, we contrast these with the estimation results to another approximation technique aforementioned, the Diagonal Super Tile (DST). Fixing $\nu_{11}=0.5$ and $\nu_{22}=1$, we vary the value of the remaining parameter responsible for spatial dependence over long distances, i.e., the range parameter, $a$. Different values of $a$ were chosen to represent weak $(a=0.03)$, moderate $(a=0.09)$, and strong $(a=0.20)$ spatial dependencies.

- Experiment 3: Predictions are made at the 2,500 prediction locations and the errors produced by the exact and the approximation models are assessed using the newly proposed multivariate MLOE/MMOM.

Fig. 14 summarizes the results of Experiment 1. The figure shows that the higher the value of the parameter responsible for the colocated dependence $(\beta)$, the lower the prediction error becomes. In bivariate datasets, the inclusion of a second variable effectively increases the number of samples available for any of the two variables when the colocated dependence between them is high (positive or negative). The more correlated $Z_{1}$ and $Z_{2}$ are, through $\beta$, the more information we get about $Z_{1}$ from $Z_{2}$, and vice versa. This echoes the theoretical results in [34], where it was shown that the colocated correlation parameter dictates the improvement introduced by cokriging (multivariate prediction) over kriging (univariate prediction). Similar conclusions were derived using other values of $a$, the parameter controlling the long range spatial dependence. This suggests that bivariate or multivariate $(p>2)$ modeling should be pursued regardless because while the gain in using a bivariate model is not so pronounced when the colocated dependence is not so high in the positive or negative direction, there is significant error reduction when the variables turn out to be highly correlated.

Fig. 13, plots the accuracy of our estimation procedure under the different TLR and exact implementations at different strengths of spatial dependence controlled by the range parameter, $a$. It also includes the results of the parameter estimation under the two different sizes of the DST. DST 

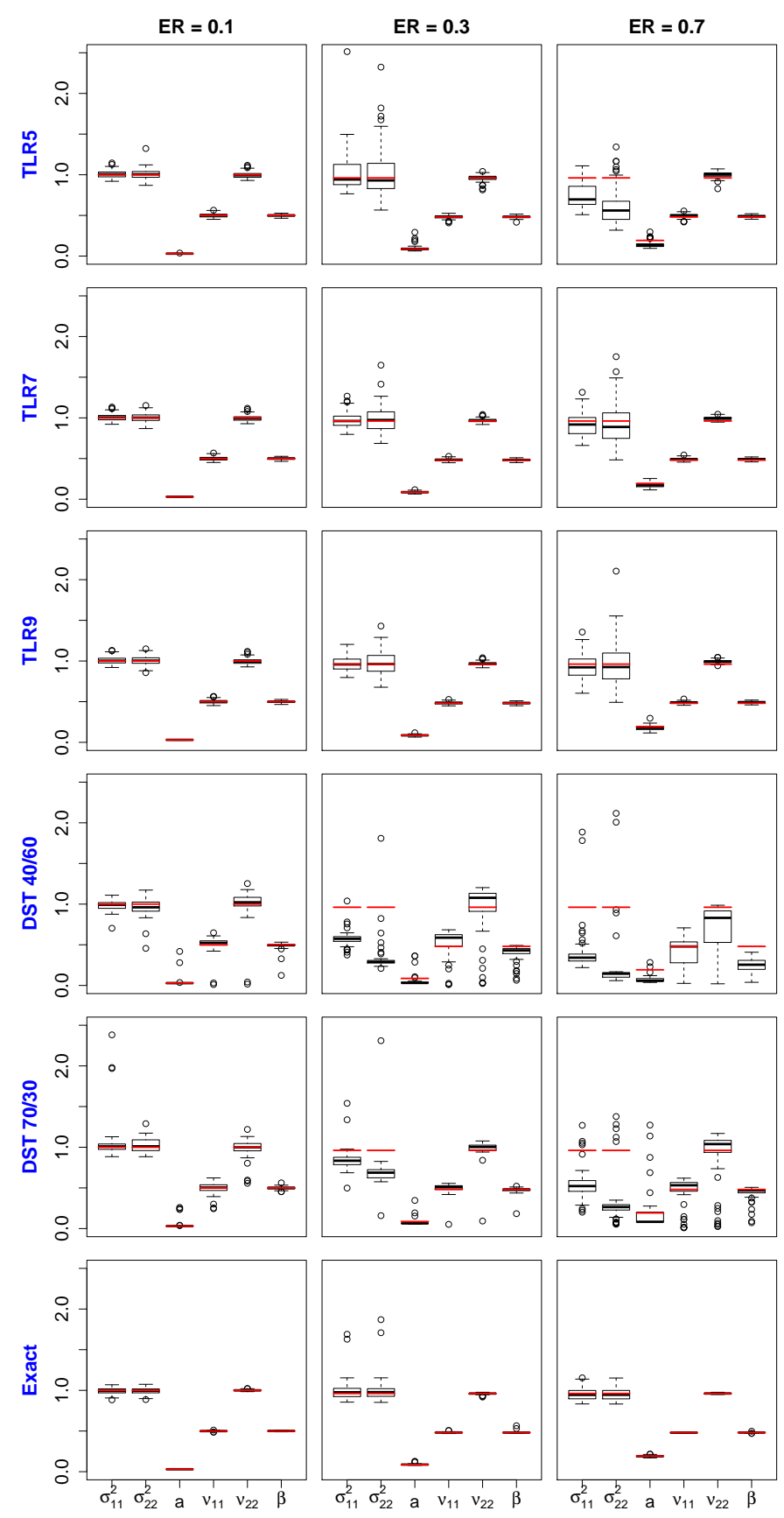

Fig. 13: Boxplots of parameter estimates under the exact, TLR, and DST implementations. The true parameters are highlighted in red. ER refers to effective range or the distance at which the marginal covariance drops to approximately 0.05 . Here $\mathrm{ER}=\{0.1,0.3,0.7\}$ corresponds to $a=\{0.03,0.09,0.2\}$.

$40 / 60$ means that $40 \%$ of the tiles from the diagonal are kept and the remaining $60 \%$ are annihilated. Similarly, DST 70/30 denotes that $70 \%$ of the tiles from the diagonal are kept and the remaining $30 \%$ are annihilated. When the spatial dependence is weak $(a=0.03)$, the boxplots across the different TLRs and the exact are identical, i.e., the medians and the standard deviations of the parameter estimates are
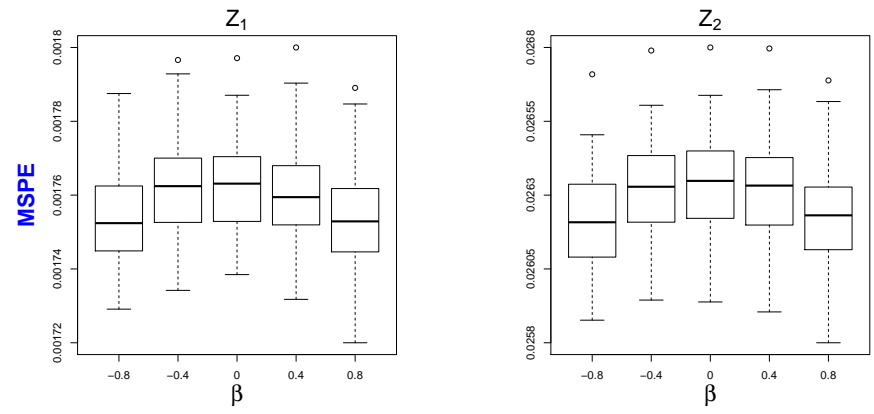

Fig. 14: Prediction error (MSPE) at different values of $\beta$ with $\sigma_{11}^{2}=\sigma_{22}^{2}=1, \nu_{11}=0.5, \nu_{22}=1$, and $a=0.09$.

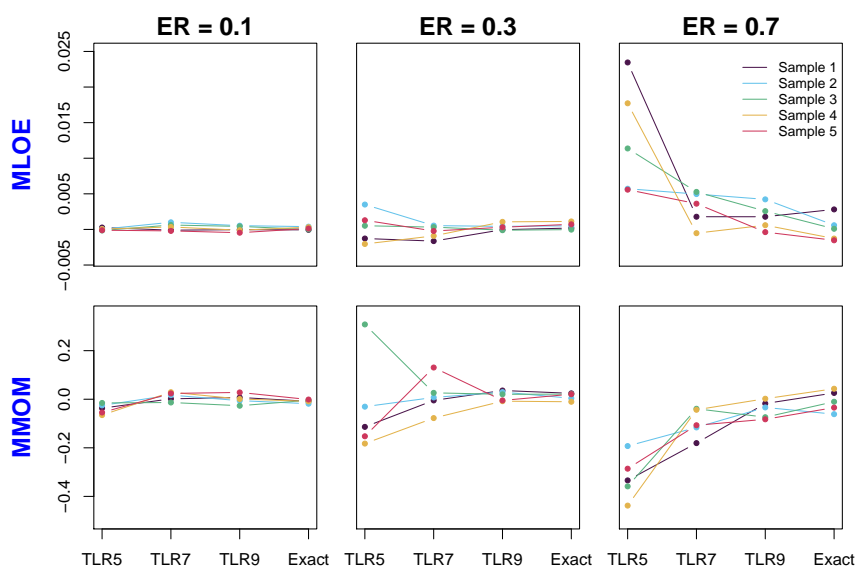

Fig. 15: Multivariate MLOE/MMOM values under exact and the different TLR models at various effective ranges.

almost equal. While the medians of the parameter estimates under DST 40/60 and DST 70/30 are close to the true parameter values, the estimates have more variability. As the dependence in space increase, i.e., $a=0.09$ (moderate) and $a=0.2$ (strong), TLR5 is insufficient and it obtains parameter estimates that are very far from the true value. While increasing the accuracy to TLR7 solves the problem for moderate spatial dependence, this level of accuracy is still inadequate for simulations with strong spatial dependence as the medians of the parameter estimates still do not coincide with the true parameter values. TLR9 remedies this problem. DST 40/60 and DST 70/30 give estimates that are far from the true values and they perform worse as the strength of spatial dependence increase. This is expected as the DST technique throws away significant amount of information in the cross-covariance matrix which is vital when the dependence in space is strong. All in all, while there are significantly more parameters to estimate in the parsimonious bivariate Matérn model, our estimation procedure can satisfactorily recover all of them. Furthermore, TLR approximation outperforms another approximation technique, i.e., the DST, and remains competitive with the exact model in terms of parameter estimation accuracy when using a higher accuracy level whenever there is stronger spatial dependence.

Using the parameter estimates in Experiment 2, we predict at the 2, 500 unsampled locations and measure the 

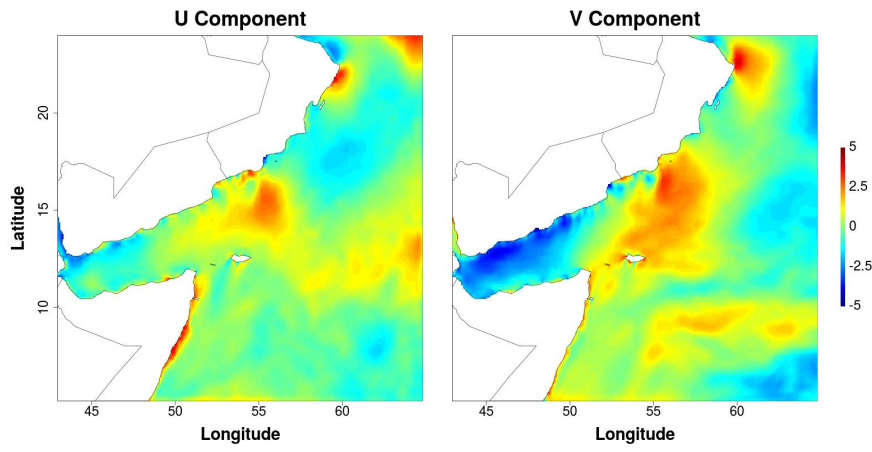

Fig. 16: Spatial images of the bivariate dataset on January 1, 2009 (after mean removal) on 116, 100 locations over the Arabian Sea.

TABLE 1: A summary of the estimated parameters and the breakdown per variable and average of the prediction error, denoted by $\mathrm{MSPE}_{i}, i=1,2$, and $\mathrm{MSPE}_{\text {avg, }}$ respectively, of the bivariate model fitted to the bivariate dataset on January 1, 2009.

Parsimonious Bivariate Matérn

\begin{tabular}{lccccc}
\hline$\hat{\sigma}_{11}^{2}$ & $\hat{\sigma}_{22}^{2}$ & $\hat{a}$ & $\hat{\nu}_{11}$ & $\hat{\nu}_{22}$ & $\hat{\beta}_{12}$ \\
\hline 0.718 & 0.710 & 0.161 & 2.283 & 2.033 & 0.192 \\
\hline & & & & \\
\cline { 2 - 4 } & $\mathrm{MSPE}_{1}$ & $\mathrm{MSPE}_{2}$ & $\mathrm{MSPE}_{\text {avg }}$ & \\
\cline { 2 - 5 } & 0.000189 & 0.000261 & 0.000225 & \\
\cline { 2 - 4 } & & & & &
\end{tabular}

multivariate MLOE/MMOM for all the TLR approximation models and the exact using Algorithm 1. Fig. 15 charts the behavior of the multivariate MLOE/MMOM of 5 randomly chosen sample bivariate random fields as we increase the accuracy levels. Commensurate to the findings in Experiment 2, bivariate random fields with higher spatial dependence necessitate TLR approximations with higher accuracy levels in order to remain competitive with the exact model.

\subsubsection{Real Data Application}

We obtained datasets with a horizontal spatial resolution of $5 \mathrm{~km}$ from a Weather Research and Forecasting (WRF) model simulation on the $\left[43^{\circ} \mathrm{E}, 65^{\circ} \mathrm{E}\right] \times\left[5^{\circ} \mathrm{S}, 24^{\circ} \mathrm{N}\right]$ region of the earth [79]. We restricted the dataset to the Arabian Sea to ensure that the measurements exhibit spatial isotropy, i.e., the cross-covariance depends only on the distance between any two locations and not on the locations themselves. Often, this isotropy assumption holds when the locations are situated in areas with similar characteristics. As the locations are all on the ocean, this behavior can be expected. The resulting subset contains $n=116,100$ locations and the two locations which are located farthest from each other have a great circle distance of $2,681 \mathrm{~km}$.

I. Bivariate Dataset

We fit the parsimonious bivariate Matérn covariance function on measurements obtained on January 1, 2009, consisting of two variables, namely, zonal wind component, $U$ (variable 1 ), and meridional wind component, $V$ (variable 2), both measured in $\mathrm{m} / \mathrm{s}$. In order to satisfy the zero-mean assumption, we remove a spatially
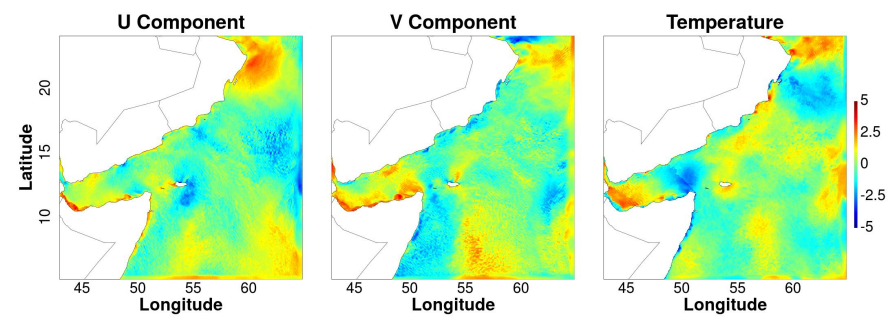

Fig. 17: Spatial images of the trivariate dataset on October 1, 2009 (after mean removal) on 116, 100 locations over the Arabian Sea.

TABLE 2: A summary of the estimated parameters and the breakdown per variable and average of the prediction error, denoted by $\mathrm{MSPE}_{i}, i=1,2,3$, and $\mathrm{MSPE}_{\text {avg, }}$, respectively, of the trivariate model fitted to the trivariate dataset on October 1, 2009.

Parsimonious Trivariate Matérn

\begin{tabular}{ccccccc}
\hline$\hat{\sigma}_{11}^{2}$ & $\hat{\sigma}_{22}^{2}$ & $\hat{\sigma}_{33}^{2}$ & $\hat{a}$ & $\hat{\nu}_{11}$ & $\hat{\nu}_{22}$ & $\hat{\nu}_{33}$ \\
\hline 0.788 & 0.874 & 0.301 & 0.0822 & 1.689 & 1.629 & 1.234 \\
\hline
\end{tabular}

\begin{tabular}{lcccccc}
\hline$\hat{\beta}_{12}$ & $\hat{\beta}_{13}$ & $\hat{\beta}_{23}$ & $\mathrm{MSPE}_{1}$ & $\mathrm{MSPE}_{2}$ & $\mathrm{MSPE}_{3}$ & $\mathrm{MSPE}_{\text {avg }}$ \\
\hline 0.243 & -0.124 & -0.059 & 0.009900 & 0.012248 & 0.021073 & 0.014407 \\
\hline
\end{tabular}

varying mean using the longitudes and latitudes as covariates. The resulting values after mean removal are approximately Gaussian and are plotted in Fig. 16 The parameter estimates for the parsimonious bivariate Matérn fitted to the dataset at $n=104,490$ observation locations are presented in Table 1 The MSPE values for the predictions done on $n_{\text {pred }}=11,610$ prediction locations are also shown. From the results, it can be seen that $U$ and $V$ (variables 1 and 2) are positively correlated since $\hat{\beta}_{12}>0$. Furthermore, the estimates of the smoothness parameters $\hat{\nu}_{11}$ and $\hat{\nu}_{22}$ suggest that $U$ and $V$ are very smooth random fields, which is certainly the case as shown in Fig. 16

II. Trivariate Dataset

We retrieve another dataset from October 1, 2009 consisting of three variables including the $U$ and $V$ wind components (in $\mathrm{m} / \mathrm{s}$ ) and temperature (in Kelvin), as variable 3, and fit a trivariate Matérn covariance function. Similarly, we remove a spatially varying mean using the longitudes and latitudes as covariates. The resulting values after mean removal are approximately Gaussian and are plotted in Fig. 17.

The parameter estimates for the parsimonious trivariate Matern are presented in Table 2 with the corresponding MSPE values. It can be seen that $U$ and $V$ (variables 1 and 2) are positively correlated while each of them are negatively correlated to variable 3 , the temperature variable. These values obtained for the correlation coefficients can be visually validated by Fig. 17. In regions where blue/green spots are observed in the $U$ and $V$ components, red/yellow spots generally occur for the temperature variable, especially in 
the $\left[53^{\circ} \mathrm{E}, 65^{\circ} \mathrm{E}\right] \times\left[13^{\circ} \mathrm{S}, 17^{\circ} \mathrm{N}\right]$ region. This inverse relationship can also be seen along the coast of Yemen and Somalia.

Note that the estimates for the smoothness and the range parameters in the bivariate model, i.e., $\hat{\nu}_{11}, \hat{\nu}_{22}$, and $\hat{a}$, are higher compared to its counterpart in the trivariate model. This is expected as the spots are larger and smoother in Fig. 16 than in Fig. 17 These foregoing results definitely show that our proposed implementation was able to successfully fit the models with physically reasonable parameter estimates.

\section{Conclusion}

We proposed a high-performance framework for modeling and inference of large spatial datasets based on the multivariate geospatial statistical modeling concept. In the context of climate and weather applications, the framework can operate on the Gaussian log-likelihood function with three (or more) associated variables, for the purpose of estimating a parameter vector, in order to predict missing measurements. Although machine learning and deep learning techniques can also be used in prediction, geospatial statistical modeling has better interpretability capabilities regarding the underlying spatial field. Both the exact and TLR-based approximation computations of the MLE operations were implemented and evaluated on large-scale experiments. The TLR-based approximation for the MLE outperformed the fully double-precision exact MLE counterpart up to 10X and 2X on different hardware architectures. Comprehensive qualitative experiments were conducted to assess the accuracy of the TLR-based estimation and prediction. We demonstrated the effectiveness of the approximation technique in achieving high performance, while preserving a convenient accuracy level. Additionally, an algorithm to compute the newly proposed multivariate MLOE/MMOM criteria was devised. This algorithm allows for the assessment of the quality of the MLE operations involving approximated models.

Future research will focus on modeling and prediction of environmental variables which are indexed in space and time. The spatial and temporal coverage of big geospatial data can be exploited to improve insights on an environmental phenomenon. Tackling the space-time problem should bring more challenges related to the problem dimension and prediction accuracy in climate/weather applications.

\section{ACKNOWLEDGEMENTS}

The authors would like to thank NVIDIA Inc., Cray Inc., and Intel Corp., the Cray Center of Excellence and Intel Parallel Computing Center awarded to the Extreme Computing Research Center (ECRC) at KAUST. For computer time, this research used GPU-based systems as well as Shaheen supercomputer, both hosted at the Supercomputing Laboratory at King Abdullah University of Science and Technology (KAUST).

\section{RefERENCES}

[1] M. Asch, T. Moore, R. Badia, M. Beck, P. Beckman, T. Bidot F. Bodin, F. Cappello, A. Choudhary, B. de Supinski, E. Deelman, J. Dongarra, A. Dubey, G. Fox, H. Fu, S. Girona, W. Gropp M. Heoux, Y. Ishikawa, K. Keahey, D. Keyes, W. Kramer, J.-F. Lavignon, Y. Lu, S. Matsuoka, B. Mohr, D. Reed, S. Requena, J. Saltz, T. Schulthess, R. Stevens, M. Swany, A. Szalay, W. Tang, G. Varoquaux, J.-P. Vilotte, R. Wisniewski, Z. Xu, and I. Zacharov, "Big data and extreme-scale computing: Pathways to convergencetoward a shaping strategy for a future software and data ecosystem for scientific inquiry," The International Journal of High Performance Computing Applications, vol. 32, no. 4, pp. 435-479, 2018

[2] X.-k. Liao, K. Lu, C.-q. Yang, J.-w. Li, Y. Yuan, M.-c. Lai, L.-b. Huang, P.-j. Lu, J.-b. Fang, J. Ren, and J. Shen, "Moving from exascale to zettascale computing: challenges and techniques," Frontiers of Information Technology E Electronic Engineering, vol. 19, no. 10, pp. 1236-1244, 2018.

[3] S. Abdulah, H. Ltaief, Y. Sun, M. G. Genton, and D. E. Keyes, "ExaGeoStat: A high performance unified software for geostatistics on manycore systems," IEEE Transactions on Parallel and Distributed Systems, vol. 29, no. 12, pp. 2771-2784, 2018.

[4] N. Doucet, H. Ltaief, D. Gratadour, and D. Keyes, "Mixedprecision tomographic reconstructor computations on hardware accelerators," in 2019 IEEE/ACM 9th Workshop on Irregular Applications: Architectures and Algorithms (IA3). IEEE, 2019, pp. 31-38.

[5] B. H. Park, H. A. Aziz, A. Morton, and R. Stewart, "High performance data driven agent-based modeling framework for simulation of commute mode choices in metropolitan area," in 2018 21st International Conference on Intelligent Transportation Systems (ITSC). IEEE, 2018, pp. 3779-3784.

[6] H.-T. Chu, "High-performance computing for measurement of cancer gene signatures," Application of Omics, AI and Blockchain in Bioinformatics Research, vol. 21, p. 109, 2019.

[7] J. Drake and I. Foster, "Introduction to the special issue on parallel computing in climate and weather modeling," Parallel Computing, vol. 21, no. 10, pp. 1539-1544, 1995.

[8] N. R. Council et al., From research to operations in weather satellites and numerical weather prediction: Crossing the valley of death. National Academies Press, 2000.

[9] "Earth Science Data Systems (ESDS) Program," January 2020, Earthdata.nasa.gov [Online].

[10] P. Burrough, "Gis and geostatistics: Essential partners for spatial analysis," Environmental and ecological statistics, vol. 8, no. 4, pp. 361-377, 2001.

[11] X. Shi, V. Kindratenko, and C. Yang, "Modern accelerator technologies for geographic information science," in Modern Accelerator Technologies for Geographic Information Science. Springer, 2013, pp. 3-6.

[12] L. Liu, A. Yang, L. Chen, W. Xiong, Q. Wu, and N. Jing, "Higiswhen gis meets hpc," in 12th International Conference on GeoComputation, Wuhan, 2013

[13] N. Stojanovic and D. Stojanovic, "High-performance computing in gis: techniques and applications," International Journal of Reasoningbased Intelligent Systems, vol. 5, no. 1, pp. 42-49, 2013.

[14] J. Zhang, "Parallel primitives-based spatial join of geospatial data on gpgpus," in Modern Accelerator Technologies for Geographic Information Science. Springer, 2013, pp. 55-67.

[15] C. Yang, K. Clarke, S. Shekhar, and C. V. Tao, "Big spatiotemporal data analytics: A research and innovation frontier," International Journal of Geographical Information Science, vol. 34, no. 6, pp. 1075$1088,2020$.

[16] J. Li, Y. Jiang, C. Yang, and Q. Huang, “Utilizing cuda-enabled gpus to support $5 \mathrm{~d}$ scientific geovisualization: A case study of simulating dust storm events," in Modern Accelerator Technologies for Geographic Information Science. Springer, 2013, pp. 69-82.

[17] X. Kang, J. Liu, C. Dong, and S. Xu, "Using high-performance computing to address the challenge of land use/land cover change analysis on spatial big data," ISPRS International Journal of GeoInformation, vol. 7, no. 7, p. 273, 2018.

[18] A.-X. Zhu, F.-H. Zhao, P. Liang, and C.-Z. Qin, "Next generation of gis: must be easy," Annals of GIS, pp. 1-16, 2020.

[19] M. Bancheri, F. Serafin, M. Bottazzi, W. Abera, G. Formetta, and R. Rigon, "The design, deployment, and testing of kriging models in GEOframe with SIK-0.9. 8," Geoscientific Model Development, vol. 11, no. 6, pp. 2189-2207, 2018. 
[20] Q. Zhang, Q. Yuan, C. Zeng, X. Li, and Y. Wei, "Missing data reconstruction in remote sensing image with a unified spatialtemporal-spectral deep convolutional neural network," IEEE Transactions on Geoscience and Remote Sensing, vol. 56, no. 8, pp. 4274-4288, 2018

[21] A. Fouilloy, C. Voyant, G. Notton, F. Motte, C. Paoli, M.-L. Nivet, E. Guillot, and J.-L. Duchaud, "Solar irradiation prediction with machine learning: Forecasting models selection method depending on weather variability," Energy, vol. 165, pp. 620-629, 2018.

[22] K. J. Bergen, P. A. Johnson, V. Maarten, and G. C. Beroza, "Machine learning for data-driven discovery in solid earth geoscience," Science, vol. 363, no. 6433, 2019.

[23] C. Zhang, I. Sargent, X. Pan, H. Li, A. Gardiner, J. Hare, and P. M. Atkinson, "Joint deep learning for land cover and land use classification," Remote Sensing of Environment, vol. 221, pp. 173187, 2019.

[24] V. Demyanov, E. Gloaguen, and M. Kanevski, "A special issue on data science for geosciences," Mathematical Geoscience, vol. 52, pp. $1-3,2020$.

[25] C. Irrgang, N. Boers, M. Sonnewald, E. A. Barnes, C. Kadow, J. Staneva, and J. Saynisch-Wagner, "Will artificial intelligence supersede earth system and climate models?" arXiv preprint arXiv:2101.09126, 2021.

[26] F. Binkowski and S. Roselle, "Models-3 community multiscale air quality (cmaq) model aerosol component 1 . model description," Journal of geophysical research: Atmospheres, vol. 108, no. D6, 2003.

[27] L. Minet, T. Chowdhury, A. Wang, Y. Gai, I. D. Posen, M. Roorda, and M. Hatzopoulou, "Quantifying the air quality and health benefits of greening freight movements," Environmental Research, vol. 183, p. 109193, 2020.

[28] S. Yu and J. Ma, "Data-driven geophysics: from dictionary learning to deep learning," arXiv preprint arXiv:2007.06183, 2020.

[29] F. Amato, F. Guignard, S. Robert, and M. Kanevski, "A novel framework for spatio-temporal prediction of environmental data using deep learning," Scientific Reports, vol. 10, no. 1, pp. 1-11, 2020.

[30] C. Kadow, D. M. Hall, and U. Ulbrich, "Artificial intelligence reconstructs missing climate information," Nature Geoscience, vol. 13, no. 6, pp. 408-413, 2020.

[31] S.-H. Kang, Y. Choi, and J. Y. Choi, "Restoration of missing patterns on satellite infrared sea surface temperature images due to cloud coverage using deep generative inpainting network," Journal of Marine Science and Engineering, vol. 9, no. 3, p. 310, 2021.

[32] J. M. McKinley and P. M. Atkinson, "A special issue on the importance of geostatistics in the era of data science," Mathematical Geoscience, vol. 52, pp. 311-315, 2020.

[33] M. G. Genton and W. Kleiber, "Cross-covariance functions for multivariate geostatistics," Statistical Science, pp. 147-163, 2015.

[34] H. Zhang and W. Cai, "When doesn't cokriging outperform kriging?" Statistical Science, pp. 176-180, 2015.

[35] D. T. Hristopulos, Random Fields for Spatial Data Modeling A Primer for Scientists and Engineers. Springer, 2020.

[36] N. Cressie and A. Zammit-Mangion, "Multivariate spatial covariance models: a conditional approach," Biometrika, vol. 103, no. 4, pp. 915-935, 2016.

[37] B. Li and H. Zhang, "An approach to modeling asymmetric multivariate spatial covariance structures," Journal of Multivariate Analysis, vol. 102, no. 10, pp. 1445-1453, 2011.

[38] K. V. Mardia and R. J. Marshall, "Maximum likelihood estimation of models for residual covariance in spatial regression," Biometrika, vol. 71, no. 1, pp. 135-146, 1984.

[39] C. G. Kaufman, M. J. Schervish, and D. W. Nychka, "Covariance tapering for likelihood-based estimation in large spatial data sets," Journal of the American Statistical Association, vol. 103, no. 484, pp. $1545-1555,2008$.

[40] K. Akbudak, H. Ltaief, A. Mikhalev, and D. Keyes, "Tile low rank Cholesky factorization for climate/weather modeling applications on manycore architectures," in Proceedings of the International Supercomputing Conference. Springer, 2017, pp. 22-40.

[41] D. E. Keyes, H. Ltaief, and G. Turkiyyah, "Hierarchical algorithms on hierarchical architectures," Philosophical Transactions of the Royal Society A, vol. 378, no. 2166, p. 20190055, 2020.

[42] S. Abdulah, H. Ltaief, Y. Sun, M. G. Genton, and D. E. Keyes, "Parallel approximation of the maximum likelihood estimation for the prediction of large-scale geostatistics simulations," in 2018 IEEE International Conference on Cluster Computing (CLUSTER). IEEE, 2018, pp. 98-108.
[43] Y. Yan and M. G. Genton, "Gaussian likelihood inference on data from trans-Gaussian random fields with Matérn covariance function," Environmetrics, vol. 29, no. 5-6, p. e2458, 2018.

[44] Y. Hong, S. Abdulah, M. G. Genton, and Y. Sun, "Efficiency assessment of approximated spatial predictions for large datasets," arXiv preprint arXiv:1911.04109, 2019.

[45] T. L. R. S. G. Goulart, L. G. Tavaresa, M. Loboscoa, R. W. dos Santosa, and F. de Oliveira Chavesb, "A parallel implementation of the ordinary kriging algorithm for heterogeneous computing environments," Journal of Computational Interdisciplinary Sciences, vol. 8, no. 3, pp. 143-152, 2017.

[46] L. Pesquer, A. Cortés, and X. Pons, "Parallel ordinary kriging interpolation incorporating automatic variogram fitting," Computers and Geosciences, vol. 37, no. 4, pp. 464-473, 2011.

[47] A. J. Rossini, L. Tierney, and N. Li, "Simple parallel statistical computing in R," Journal of Computational and Graphical Statistics, vol. 16, no. 2, pp. 399-420, 2007.

[48] T. Cheng, "Accelerating universal kriging interpolation algorithm using cuda-enabled GPU," Computers and Geosciences, vol. 54, no. 2 pp. 178-183, 2013.

[49] P. Tahmasebi, M. Sahimi, G. Mariethoz, and A. Hezarkhani, "Accelerating geostatistical simulations using graphics processing units (GPU)," Computers \& Geosciences, vol. 46, pp. 51-59, 2012.

[50] V. Allombert, D. Michea, F. Dupros, C. Bellier, B. Bourgine, H. Aochi, and S. Jubertie, "An out-of-core GPU approach for accelerating geostatistical interpolation," Procedia Computer Science, vol. 29, pp. 888-896, 2014.

[51] W. Zhuo, C. Paciorek, C. Kaufman, W. Bethel et al., "Parallel kriging analysis for large spatial datasets," in 2011 IEEE 11th International Conference on Data Mining Workshops. IEEE, 2011, pp. 38-44.

[52] S. Abdulah, Y. Li, J. Cao, H. Ltaief, D. E. Keyes, M. G. Genton, and Y. Sun, "ExaGeoStatR: A package for large-scale geostatistics in R," arXiv preprint arXiv:1908.06936, 2019.

[53] "The Chameleon project: A dense linear algebra software for heterogeneous architectures," May 2020, available at https://project. inria.fr/chameleon/

[54] S. Abdulah, K. Akbudak, W. Boukaram, A. Charara, D. Keyes, H. Ltaief, A. Mikhalev, D. Sukkari, and G. Turkiyyah, "Hierarchical computations on manycore architectures (HiCMA)," See http://github. com/ecrc/hicma, 2019.

[55] C. Augonnet, S. Thibault, R. Namyst, and P.-A. Wacrenier, "StarPU: a unified platform for task scheduling on heterogeneous multicore architectures," Concurrency and Computation: Practice and Experience, vol. 23, no. 2, pp. 187-198, 2011.

[56] T. Gneiting, W. Kleiber, and M. Schlather, "Matérn crosscovariance functions for multivariate random fields," Journal of the American Statistical Association, vol. 105, no. 491, pp. 1167-1177, 2010.

[57] T. V. Apanasovich, M. G. Genton, and Y. Sun, "A valid Matérn class of cross-covariance functions for multivariate random fields with any number of components," Journal of the American Statistical Association, vol. 107, no. 497, pp. 180-193, 2012.

[58] S. Banerjee, A. E. Gelfand, A. O. Finley, and H. Sang, "Gaussian predictive process models for large spatial data sets," Journal of the Royal Statistical Society: Series B (Statistical Methodology), vol. 70, no. 4, pp. 825-848, 2008.

[59] M. Katzfuss, "A multi-resolution approximation for massive spatial datasets," Journal of the American Statistical Association, vol. 112, no. 517, pp. 201-214, 2017.

[60] H. Huang and Y. Sun, "Hierarchical low rank approximation of likelihoods for large spatial datasets," Journal of Computational and Graphical Statistics, vol. 27, no. 1, pp. 110-118, 2018.

[61] R. Furrer, M. G. Genton, and D. Nychka, "Covariance tapering for interpolation of large spatial datasets," Journal of Computational and Graphical Statistics, vol. 15, no. 3, pp. 502-523, 2006.

[62] H. Rue, S. Martino, and N. Chopin, "Approximate Bayesian inference for latent Gaussian models by using integrated nested Laplace approximations," Journal of the Royal Statistical Society: Series B (statistical methodology), vol. 71, no. 2, pp. 319-392, 2009.

[63] S. Banerjee, "High-dimensional Bayesian Geostatistics," Bayesian analysis, vol. 12, no. 2, p. 583, 2017.

[64] "The STARS-H project: Software for testing accuracy, reliability and scalability of hierarchical computations," May 2020, available at https://github.com/ecrc/stars-h

[65] S. G. Johnson, "The NLopt nonlinear-optimization package," 2014 http://ab-initio.mit.edu/nlopt 
[66] A. Yarkhan, J. Kurzak, and J. Dongarra, "Quark users guide," Electrical Engineering and Computer Science, Innovative Computing Laboratory, University of Tennessee, vol. 268, 2011.

[67] G. Bosilca, A. Bouteiller, A. Danalis, M. Faverge, T. Hérault, and J. J. Dongarra, "Parsec: Exploiting heterogeneity to enhance scalability," Computing in Science \& Engineering, vol. 15, no. 6, pp. 36-45, 2013.

[68] T. A. Severini, Likelihood Methods in Statistics. Oxford University Press, 2000

[69] A. Buttari, J. Langou, J. Kurzak, and J. Dongarra, "A class of parallel tiled linear algebra algorithms for multicore architectures," Parallel Computing, vol. 35, no. 1, pp. 38-53, 2009.

[70] K. Akbudak, H. Ltaief, A. Mikhalev, A. Charara, A. Esposito, and D. Keyes, "Exploiting data sparsity for large-scale matrix computations," in European Conference on Parallel Processing. Springer, 2018, pp. 721-734.

[71] G. Morton, A Computer Oriented Geodetic Data Base and a New Technique in File Sequencing. International Business Machines Company, New York, 1966.

[72] Q. Cao, Y. Pei, K. Akbudak, A. Mikhalev, G. Bosilca, H. Ltaief, D. Keyes, and J. Dongarra, "Extreme-Scale Task-Based Cholesky Factorization Toward Climate and Weather Prediction Applications," in Proceedings of the Platform for Advanced Scientific Computing Conference. ACM, 2020.

[73] T. Mary, "Block Low-Rank Multifrontal Solvers: Complexity, Performance, and Scalability," Ph.D. dissertation, Paul Sabatier University, Toulouse, France, November 2017.

[74] S. Ambikasaran and E. Darve, "An $\mathcal{O}(N \log N)$ fast direct solver for partial Hierarchically Semiseparable matrices," Journal of Scientific Computing, vol. 57, no. 3, pp. 477-501, 2013.

[75] A. Aminfar, S. Ambikasaran, and E. Darve, "A fast block low-rank dense solver with applications to finite-element matrices," Journal of Computational Physics, vol. 304, pp. 170-188, 2016.

[76] W. Hackbusch, Hierarchical Matrices: Algorithms and Analysis. Springer, 2015, vol. 49

[77] E. Anderson, J. Dongarra, and S. Ostrouchov, "LAPACK working note 41: Installation guide for LAPACK, University of Tennessee," CS-92-151, February 1992 (revised June 1999), Tech. Rep., 1992.

[78] "Stream:he de facto industry standard benchmark for measuring sustained memory bandwidth," Jan 2021, available at https: //github.com/jeffhammond/STREAM

[79] C. Yip, "Statistical characteristics and mapping of near-surface and elevated wind resources in the Middle East," Ph.D. dissertation, King Abdullah University of Science and Technology, 2018.

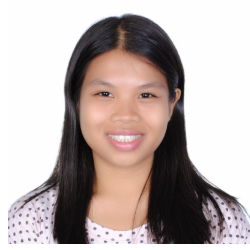

Mary Lai Salvaña is a $\mathrm{PhD}$ student in the Spatio-Temporal Statistics \& Data Science group at King Abdullah University of Science and Technology (KAUST). She received her BS and MS degrees in Applied Mathematics in 2015 and 2016 from Ateneo de Manila University, Philippines. Her research interest includes multivariate spatio-temporal statistics and highperformance computing for large spatial and spatio-temporal datasets.

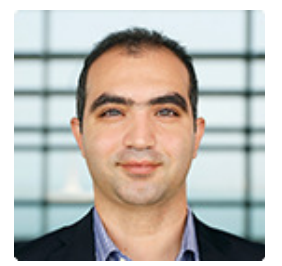

Sameh Abdulah is a research scientist at the Extreme Computing Research Center, King Abdullah University of Science and Technology, Saudi Arabia. Sameh received his MS and PhD degrees from Ohio State University, Columbus, USA, in 2014 and 2016, His work is centered around High Performance Computing (HPC) applications, bitmap indexing in big data, large spatial datasets, parallel statistical applications, algorithm-based fault tolerance, and Machine Learning and Data Mining algorithms.

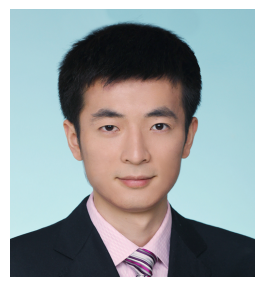

Huang Huang is a research scientist in the Spatio-Temporal Statistics \& Data Science group at King Abdullah University of Science and Technology (KAUST). Before working at KAUST, he did research on statistical computing for climate applications as a postdoc at the National Center for Atmospheric Research (NCAR), the Statistical and Applied Mathematical Sciences Institute (SAMSI), and Duke University. He received his Ph.D. in Statistics in 2017 from KAUST, master and bachelor in Mathematics in 2014 and 2011 from Fudan University. His research interest includes spatio-temporal statistics, functional data analysis, Bayesian modeling, machine learning, and high-performance computing for large datasets.

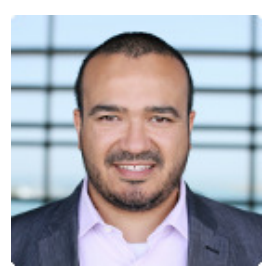

Hatem Ltaief is the Principal Research Scientist in the Extreme Computing Research Center at King Abdullah University of Science and Technology, Saudi Arabia. His research interests include parallel numerical algorithms, fault tolerant algorithms, parallel programming models, and performance optimizations for multicore architectures and hardware accelerators. His current research collaborators include Aramco, Total, Observatoire de Paris, NVIDIA, and Intel.

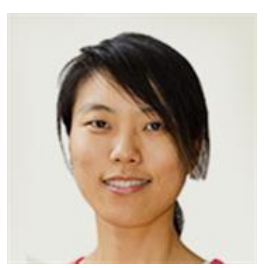

Ying Sun received the $\mathrm{PhD}$ degree in statistics from Texas A\&M University in 2011. She is an associate professor of statistics with the King Abdullah University of Science and Technology (KAUST) in Saudi Arabia. Her research interests include spatio-temporal statistics with environmental applications, computational methods for large datasets, uncertainty quantification and visualization, functional data analysis, robust statistics, and statistics of extremes.

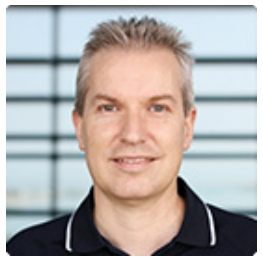

Marc Genton received the PhD degree in statistics (1996) from the Swiss Federal Institute of Technology (EPFL), Lausanne. He is a distinguished professor of statistics with the King Abdullah University of Science and Technology (KAUST) in Saudi Arabia. He is a fellow of the American Statistical Association, of the Institute of Mathematical Statistics, and the American Association for the Advancement of Science, and is an elected member of the International Statistical Institute. His research interests include statistical analysis, flexible modeling, prediction, and uncertainty quantification of spatio-temporal data, with applications in environmental and climate science, renewable energies, geophysics, and marine science.

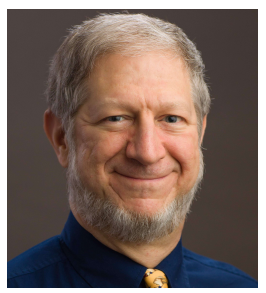

David Keyes received the BSE degree in aerospace and mechanical sciences from Princeton University, in 1978, and the PhD degree in applied mathematics from Harvard University, in 1984. He directs the Extreme Computing Research Center, KAUST. He works at the interface between parallel computing and the numerical analysis of PDEs, with a focus on scalable implicit solvers. He helped develop and popularize the Newton-Krylov-Schwarz (NKS), Additive Schwarz Preconditioned Inexact Newton (ASPIN), and Algebraic Fast Multipole (AFM) methods. He is a fellow of the SIAM, AMS, and AAAS. 